\title{
Supersymmetric RG flows and Janus from type II orbifold compactification
}

\author{
Parinya Karndumri ${ }^{\mathrm{a}}$, Khem Upathambhakul ${ }^{\mathrm{b}}$ \\ String Theory and Supergravity Group, Department of Physics, Faculty of Science, Chulalongkorn University, 254 Phayathai Road, Pathumwan, \\ Bangkok 10330, Thailand
}

Received: 28 April 2017 / Accepted: 23 June 2017 / Published online: 7 July 2017

(C) The Author(s) 2017. This article is an open access publication

\begin{abstract}
We study holographic RG flow solutions within four-dimensional $N=4$ gauged supergravity obtained from type IIA and IIB string theories compactified on $T^{6} / \mathbb{Z}_{2} \times \mathbb{Z}_{2}$ orbifold with gauge, geometric and non-geometric fluxes. In type IIB non-geometric compactifications, the resulting gauged supergravity has $I S O(3) \times I S O(3)$ gauge group and admits an $N=4 \mathrm{AdS}_{4}$ vacuum dual to an $N=4$ superconformal field theory (SCFT) in three dimensions. We study various supersymmetric RG flows from this $N=4$ SCFT to $N=4$ and $N=1$ non-conformal field theories in the IR. The flows preserving $N=4$ supersymmetry are driven by relevant operators of dimensions $\Delta=1,2$ or alternatively by one of these relevant operators, dual to the dilaton, and irrelevant operators of dimensions $\Delta=4$ while the $N=1$ flows in addition involve marginal deformations. Most of the flows can be obtained analytically. We also give examples of supersymmetric Janus solutions preserving $N=4$ and $N=1$ supersymmetries. These solutions should describe two-dimensional conformal defects within the dual $N=4$ SCFT. Geometric compactifications of type IIA theory give rise to $N=4$ gauged supergravity with $I S O(3) \ltimes U(1)^{6}$ gauge group. In this case, the resulting gauged supergravity admits an $N=1 \mathrm{AdS}_{4}$ vacuum. We also numerically study possible $N=1 \mathrm{RG}$ flows to non-conformal field theories in this case.
\end{abstract}

\section{Introduction}

Along the line of research within the context of the AdS/CFT correspondence, the study of holographic RG flows is of particular interest since the original proposal in [1]. There is much work exploring this type of holographic solutions in various space-time dimensions with different numbers of

\footnotetext{
a e-mail: parinya.ka@hotmail.com

be-mail: keima.tham@gmail.com
}

supersymmetry. In this paper, we will particularly consider holographic RG flows within three-dimensional superconformal field theories (SCFTs) using gauged supergravity in four dimensions. This might give some insight to the dynamics of strongly coupled SCFTs in three dimensions and related brane configurations in string/M-theory.

Most of the previously studied holographic RG flows have been found within the maximal $N=8$ gauged supergravities [2-9]. Many of these solutions describe various deformations of the $N=8$ SCFTs arising from M2-brane world-volume proposed in $[10,11]$. Similar study in the case of lower number of supersymmetry is, however, less known. For example, a number of RG flow solutions have appeared only recently in $N=3$ and $N=4$ gauged supergravities [12-14]. In this work, we will give more solutions of this type from the half-maximal $N=4$ gauged supergravity.

$N=4$ supergravity allows for coupling to an arbitrary number of vector multiplets. With $n$ vector multiplets, there are $6 n+2$ scalars, 2 from gravity and $6 n$ from vector multiplets, parametrized by $S L(2, \mathbb{R}) / S O(2) \times$ $S O(6, n) / S O(6) \times S O(n)$ coset. The $N=4$ gauged supergravity has been constructed for a long time in $[15,16]$. Gaugings constructed in [15] are called electric gaugings since only electric $n+6$ vector fields appearing in the ungauged Lagrangian gauge a subgroup of $S O(6, n)$. These vector fields transform as a fundamental representation of $S O(6, n)$. The scalar potential of the resulting gauged supergravity constructed in this way does not possess any $\mathrm{AdS}_{4}$ critical points $[17,18]$. This is not the case for the construction in [16] in which non-trivial $S L(2, \mathbb{R})$ phases have been included.

The most general gauging in which both electric vector fields and their magnetic dual can participate has been constructed in [19] using the embedding tensor formalism. A general gauge group is a subgroup of the full duality group $S L(2, \mathbb{R}) \times S O(6, n)$ with the vector fields and their magnetic dual transforming as a doublet of $S L(2, \mathbb{R})$. In this 
work, we will consider $N=4$ gauged supergravity obtained from compactifications of type II string theories with various fluxes given in [20]; for other work along this line, see for example [21-23].

In [20], the scalar potential arising from flux compactifications of type IIA and IIB theories on $T^{6} / \mathbb{Z}_{2} \times \mathbb{Z}_{2}$ within a truncation to $S O(3)$ singlet scalars has been considered, and some $\mathrm{AdS}_{4}$ critical points together with their properties have been given. For type IIB non-geometric compactification, the vacuum structure is very rich even with only a few number of fluxes turned on. Among these vacua, there exists an $N=4 \mathrm{AdS}_{4}$ critical point dual to an $N=4$ SCFT with $S O(3) \times S O(3)$ global symmetry. In addition, the full classification of vacua from type IIA geometric compactification has also been given. In this case, there exist a number of stable non-supersymmetric $\mathrm{AdS}_{4}$ critical points as well as an $N=1 \mathrm{AdS}_{4}$ vacuum; see [24] for an $N=1$ supersymmetric $\mathrm{AdS}_{4}$ vacuum in massive type IIA theory.

We are particularly interested in $N=4$ and $N=1 \mathrm{AdS}_{4}$ critical points from these two compactifications. They correspond to $N=4$ and $N=1$ SCFTs in three dimensions with global symmetries $S O(3) \times S O(3)$ and $S O(3)$, respectively. We will look for possible supersymmetric deformations within these two SCFTs in the form of RG flows to non-conformal phases preserving some amount of supersymmetry. These deformations are described by supersymmetric domain walls in the $N=4$ gauged supergravity. In the case of $N=1$ SCFT arising from massive type IIA theory, nonsupersymmetric RG flows to conformal fixed points in the IR have been recently found in [25].

For type IIB compactification, we will also consider supersymmetric Janus solutions describing $(1+1)$-dimensional conformal interfaces in the $N=4$ SCFT. This type of solutions breaks conformal symmetry in three dimensions but preserves a smaller conformal symmetry on the lowerdimensional interface. Similar to the RG flow solutions, there are only a few examples of these solutions within the context of four-dimensional gauged supergravities [14,26-28], see also [29-31] for examples of higher-dimensional solutions. They also play an important role in the holographic study of interface and boundary CFTs [32,33]. We will give more examples of these solutions in $N=4$ gauged supergravity obtained from non-geometric flux compactification.

The paper is organized as follows. In Sect. 2, we review relevant formulas and introduce some notations for $N=4$ gauged supergravity in the embedding tensor formalism. In Sects. 3 and 4, we give a detailed analysis of supersymmetric RG flow and Janus solutions obtained from non-geometric type IIB compactification. Similar study of RG flows from geometric type IIA compactification will be given in Sect. 5. We finally give some conclusions and comments on the results in Sect. 6. We have also included an Appendix con- taining more details on the conventions and the explicit form of complicated equations.

\section{$2 N=4$ gauged supergravity coupled to six vector multiplets}

We first review relevant information and necessary formulas of four-dimensional $N=4$ gauged supergravity which is the framework we use to find supersymmetric solutions. We mainly follow the most general gauging of $N=4$ supergravity in the embedding tensor formalism given in [19] in which more details on the construction can be found. $N=4$ supersymmetry allows for coupling the supergravity multiplet to an arbitrary number of vector multiplets. We will begin with a general formulation of $N=4$ gauged supergravity with $n$ vector multiplets and later specify to the case of six vector multiplets.

In half-maximal $N=4$ supergravity, the supergravity multiplet consists of the graviton $e_{\mu}^{\hat{\mu}}$, four gravitini $\psi_{\mu}^{i}$, six vectors $A_{\mu}^{m}$, four spin- $\frac{1}{2}$ fields $\chi^{i}$ and one complex scalar $\tau$ consisting of the dilaton $\phi$ and the axion $\chi$. The complex scalar can be parametrized by $S L(2, \mathbb{R}) / S O(2)$ coset. The supergravity multiplet can couple to an arbitrary number $n$ of vector multiplets, and each vector multiplet contains a vector field $A_{\mu}$, four gaugini $\lambda^{i}$ and six scalars $\phi^{m}$. Similar to the dilaton and the axion in the gravity multiplet, the $6 n$ scalar fields in these vector multiplets can be parametrized by $S O(6, n) / S O(6) \times S O(n)$ coset.

We will use the following convention on various indices appearing throughout the paper. Space-time and tangent space indices are denoted, respectively, by $\mu, v, \ldots=$ $0,1,2,3$ and $\hat{\mu}, \hat{v}, \ldots=0,1,2,3$. The $S O(6) \sim S U(4) \mathrm{R}-$ symmetry indices will be described by $m, n=1, \ldots, 6$ for the $S O(6)$ vector representation and $i, j=1,2,3,4$ for the $S O(6)$ spinor or $S U$ (4) fundamental representation. The $n$ vector multiplets will be labeled by indices $a, b=1, \ldots, n$. All fields in the vector multiplets then carry an additional index in the form of $\left(A_{\mu}^{a}, \lambda^{i a}, \phi^{m a}\right)$. Fermionic fields and the supersymmetry parameters transform in the fundamental representation of $S U(4)_{R} \sim S O(6)_{R}$ R-symmetry and are subject to the chirality projections

$\gamma_{5} \psi_{\mu}^{i}=\psi_{\mu}^{i}, \quad \gamma_{5} \chi^{i}=-\chi^{i}, \quad \gamma_{5} \lambda^{i}=\lambda^{i}$

On the other hand, for the fields transforming in the antifundamental representation of $S U(4)_{R}$, we have

$\gamma_{5} \psi_{\mu i}=-\psi_{\mu i}, \quad \gamma_{5} \chi_{i}=\chi_{i}, \quad \gamma_{5} \lambda_{i}=-\lambda_{i}$.

Gaugings of the matter-coupled $N=4$ supergravity can be efficiently described by using the embedding tensor. This tensor encodes all the information as regards the 
embedding of any gauge group $G_{0}$ in the global or duality symmetry group $G=S L(2, \mathbb{R}) \times S O(6, n)$ in a $G$ covariant way. According to the analysis in [19], a general gauging can be described by two components of the embedding tensor $\xi^{\alpha M}$ and $f_{\alpha M N P}$ with $\alpha=(+,-)$ and $M, N=(m, a)=1, \ldots, n+6$ denoting fundamental representations of $S L(2, \mathbb{R})$ and $S O(6, n)$, respectively. The electric vector fields $A^{+M}=\left(A_{\mu}^{m}, A_{\mu}^{a}\right)$, appearing in the ungauged Lagrangian, and their magnetic dual $A^{-M}$ form a doublet under $S L(2, \mathbb{R})$ denoted by $A^{\alpha M}$. A particular electric-magnetic frame in which the $S O(2) \times S O(6, n)$ symmetry, with $S O(2) \subset S L(2, \mathbb{R})$, is manifest in the action can always be chosen. In this frame, $A^{+M}$ and $A^{-M}$ have charges +1 and -1 under this $S O(2)$.

In general, a subgroup of both $S L(2, \mathbb{R})$ and $S O(6, n)$ can be gauged, and the magnetic vector fields can also participate in the gauging. Furthermore, it has been shown in [17], see also [18], that purely electric gaugings do not admit $\mathrm{AdS}_{4}$ vacua unless an $S L(2, \mathbb{R})$ phase is included [16]. The latter is, however, incorporated in the magnetic component $f_{-M N P}$ [19]. Accordingly, we will consider only gaugings involving both electric and magnetic vector fields in order to obtain $\mathrm{AdS}_{4}$ vacua. We will see that gauged supergravities obtained from type II compactifications are precisely of this form.

The gauge covariant derivative can be written as

$D_{\mu}=\nabla_{\mu}-g A_{\mu}^{\alpha M} \Theta_{\alpha M}{ }^{N P} t_{N P}+g A_{\mu}^{M(\alpha} \epsilon^{\beta) \gamma_{\xi}} \xi_{\gamma} t_{\alpha \beta}$

where $\nabla_{\mu}$ is the usual space-time covariant derivative including the spin connection. $t_{M N}$ and $t_{\alpha \beta}$ are $S O(6, n)$ and $S L(2, \mathbb{R})$ generators which can be chosen as

$\left(t_{M N}\right)_{P}{ }^{Q}=2 \delta_{[M}^{Q} \eta_{N] P}, \quad\left(t_{\alpha \beta}\right)_{\gamma}{ }^{\delta}=2 \delta_{(\alpha}^{\delta} \epsilon_{\beta) \gamma}$

with $\epsilon^{\alpha \beta}=-\epsilon^{\beta \alpha}$ and $\epsilon^{+-}=1 . \eta_{M N}=\operatorname{diag}(-1,-1,-1$, $-1,-1,-1,1, \ldots, 1)$ is the $S O(6, n)$ invariant tensor, and $g$ is the gauge coupling constant that can be absorbed in the embedding tensor $\Theta$.

The embedding tensor component $\Theta_{\alpha M N P}$ can be written in terms of $\xi^{\alpha M}$ and $f_{\alpha M N P}$ components as

$\Theta_{\alpha M N P}=f_{\alpha M N P}-\xi_{\alpha[N} \eta_{P] M}$.

To define a consistent gauging, the embedding tensor has to satisfy a quadratic constraint. This ensures that the gauge generators

$X_{\alpha M}=\Theta_{\alpha M N P} t^{N P}-\xi_{M}^{\beta} t_{\alpha \beta}$

form a closed algebra.

In this work, we will consider solutions with only the metric and scalars non-vanishing. In addition, we will consider gaugings with only $f_{\alpha M N P}$ non-vanishing. Therefore, we will set all vector fields and $\xi_{\alpha M}$ to zero from now on. In particular, this simplifies the full quadratic constraint to

$f_{\alpha R[M N} f_{\beta P Q]}^{R}=0, \quad \epsilon^{\alpha \beta} f_{\alpha M N R} f_{\beta P Q}{ }^{R}=0$.

For electric gaugings, these relations reduce to the usual Jacobi identity for $f_{M N P}=f_{+M N P}$ as shown in $[15,16]$.

The scalar coset manifold $S L(2, \mathbb{R}) / S O(2) \times S O(6, n) /$ $S O(6) \times S O(n)$ can be described by the coset representative $\left(\mathcal{V}_{\alpha}, \mathcal{V}_{M}{ }^{A}\right)$ with $A=(m, a)$. The first factor can be parametrized by

$\mathcal{V}_{\alpha}=\frac{1}{\sqrt{\operatorname{Im} \tau}}\left(\begin{array}{l}\tau \\ 1\end{array}\right)$

or equivalently by a symmetric $2 \times 2$ matrix

$M_{\alpha \beta}=\operatorname{Re}\left(\mathcal{V}_{\alpha} \mathcal{V}_{\beta}^{*}\right)=\frac{1}{\operatorname{Im} \tau}\left(\begin{array}{cc}|\tau|^{2} & \operatorname{Re} \tau \\ \operatorname{Re} \tau & 1\end{array}\right)$.

Note also that $\operatorname{Im}\left(\mathcal{V}_{\alpha} \mathcal{V}_{\beta}^{*}\right)=\epsilon_{\alpha \beta}$. The complex scalar $\tau$ can also be written in terms of the dilaton $\phi$ and the axion $\chi$ as

$\tau=\chi+i e^{\phi}$.

For the $S O(6, n) / S O(6) \times S O(n)$ factor, we introduce another coset representative $\mathcal{V}_{M}{ }^{A}$ transforming by left and right multiplications under $S O(6, n)$ and $S O(6) \times S O(n)$, respectively. From the splitting of the index $A=(m, a)$, we can write the coset representative as $\mathcal{V}_{M}{ }^{A}=\left(\mathcal{V}_{M}{ }^{m}, \mathcal{V}_{M}{ }^{a}\right)$. Being an element of $S O(6, n)$, the matrix $\mathcal{V}_{M}{ }^{A}$ satisfies the relation

$\eta_{M N}=-\mathcal{V}_{M}{ }^{m} \mathcal{V}_{N}{ }^{m}+\mathcal{V}_{M}{ }^{a} \mathcal{V}_{N}{ }^{a}$

As in the $S L(2, \mathbb{R}) / S O(2)$ factor, we can parametrize the $S O(6, n) / S O(6) \times S O(n)$ coset in terms of a symmetric matrix

$M_{M N}=\mathcal{V}_{M}{ }^{m} \mathcal{V}_{N}{ }^{m}+\mathcal{V}_{M}{ }^{a} \mathcal{V}_{N}{ }^{a}$

We are now in a position to give the bosonic Lagrangian with the vector fields and auxiliary two-form fields vanishing

$e^{-1} \mathcal{L}=\frac{1}{2} R+\frac{1}{16} \partial_{\mu} M_{M N} \partial^{\mu} M^{M N}-\frac{1}{4(\operatorname{Im} \tau)^{2}} \partial_{\mu} \tau \partial^{\mu} \tau^{*}-V$

where $e$ is the vielbein determinant. The scalar potential can be written in terms of scalar coset representatives and the embedding tensor as

$V=\frac{g^{2}}{16}\left[f_{\alpha M N P} f_{\beta Q R S} M^{\alpha \beta}\right.$ 


$$
\begin{aligned}
& \times\left[\frac{1}{3} M^{M Q} M^{N R} M^{P S}+\left(\frac{2}{3} \eta^{M Q}-M^{M Q}\right) \eta^{N R} \eta^{P S}\right] \\
& \left.-\frac{4}{9} f_{\alpha M N P} f_{\beta Q R S} \epsilon^{\alpha \beta} M^{M N P Q R S}\right]
\end{aligned}
$$

where $M^{M N}$ is the inverse of $M_{M N}$, and $M^{M N P Q R S}$ is defined by

$M_{M N P Q R S}=\epsilon_{\text {mnpqrs }} \mathcal{V}_{M}{ }^{m} \mathcal{V}_{N}{ }^{n} \mathcal{V}_{P}{ }^{p} \mathcal{V}_{Q}{ }^{q} \mathcal{V}_{R}{ }^{r} \mathcal{V}_{S}{ }^{s}$

with indices raised by $\eta^{M N}$.

Before giving an explicit parametrization of the scalar coset, we give fermionic supersymmetry transformations of $N=4$ gauged supergravity which play an important role in subsequent analyses. These are given by

$$
\begin{aligned}
\delta \psi_{\mu}^{i} & =2 D_{\mu} \epsilon^{i}-\frac{2}{3} g A_{1}^{i j} \gamma_{\mu} \epsilon_{j}, \\
\delta \chi^{i} & =i \epsilon^{\alpha \beta} \mathcal{V}_{\alpha} D_{\mu} \mathcal{V}_{\beta} \gamma^{\mu} \epsilon^{i}-\frac{4}{3} i g A_{2}^{i j} \epsilon_{j}, \\
\delta \lambda_{a}^{i} & =2 i \mathcal{V}_{a}{ }^{M} D_{\mu} \mathcal{V}_{M}{ }^{i j} \gamma^{\mu} \epsilon_{j}+2 i g A_{2 a j}{ }^{i} \epsilon^{j} .
\end{aligned}
$$

The fermion shift matrices, appearing in fermionic mass-like terms in the gauged Lagrangian, are defined by

$$
\begin{aligned}
A_{1}^{i j} & =\epsilon^{\alpha \beta}\left(\mathcal{V}_{\alpha}\right)^{*} \mathcal{V}_{k l}{ }^{M} \mathcal{V}_{N}{ }^{i k} \mathcal{V}_{P}{ }^{j l} f_{\beta M}{ }^{N P}, \\
A_{2}^{i j} & =\epsilon^{\alpha \beta} \mathcal{V}_{\alpha} \mathcal{V}_{k l}{ }^{M} \mathcal{V}_{N}{ }^{i k} \mathcal{V}_{P}{ }^{j l} f_{\beta M}{ }^{N P}, \\
A_{2 a i}{ }^{j} & =\epsilon^{\alpha \beta} \mathcal{V}_{\alpha} \mathcal{V}^{M}{ }_{a} \mathcal{V}^{N}{ }_{i k} \mathcal{V}_{P}{ }^{j k} f_{\beta M N}{ }^{P}{ }^{P}
\end{aligned}
$$

where $\mathcal{V}_{M}{ }^{i j}$ is defined in terms of the 't Hooft symbols $G_{m}^{i j}$ and $\mathcal{V}_{M}{ }^{m}$ as

$\mathcal{V}_{M}{ }^{i j}=\frac{1}{2} \mathcal{V}_{M}{ }^{m} G_{m}^{i j}$

and similarly for its inverse

$\mathcal{V}^{M}{ }_{i j}=-\frac{1}{2} \mathcal{V}_{m}^{M}\left(G_{m}^{i j}\right)^{*}$.

$G_{m}^{i j}$ convert an index $m$ in vector representation of $S O(6)$ to an anti-symmetric pair of indices $[i j]$ in the $S U(4)$ fundamental representation. They satisfy the relations

$G_{m i j}=-\left(G_{m}^{i j}\right)^{*}=-\frac{1}{2} \epsilon_{i j k l} G_{m}^{k l}$.

The explicit form of these matrices can be found in the appendix.

We finally note the expression for the scalar potential written in terms of $A_{1}$ and $A_{2}$ tensors as

$V=-\frac{1}{3} A_{1}^{i j} A_{1 i j}+\frac{1}{9} A_{2}^{i j} A_{2 i j}+\frac{1}{2} A_{2 a i}{ }^{j} A_{2 a j}{ }^{i}$
It follows that unbroken supersymmetry corresponds to an eigenvalue of $A_{1}^{i j}, \alpha$, satisfying $V_{0}=-\frac{\alpha^{2}}{3}$ where $V_{0}$ is the value of the scalar potential at the vacuum, the cosmological constant.

We now consider the case of $n=6$ vector multiplets. Possible gauge groups are accordingly subgroups of $S O(6,6)$ for $\xi_{\alpha M}=0$. Following [20], we restrict ourselves to solutions preserving at least $S O(3)$ subgroup of the full gauge group. The residual $S O(3)$ symmetry is embedded in $S O(6,6)$ as a diagonal subgroup of $S O(3) \times S O(3) \times$ $S O(3) \times S O(3)$ with the four factors of $S O(3)$ being subgroups of $S O(6) \times S O(6) \subset S O(6,6)$. The 36 scalars within $S O(6,6) / S O(6) \times S O(6)$ coset transform as $(\mathbf{6}, \mathbf{6})$ under $S O(6) \times S O(6)$ compact subgroup. The above embedding of $S O(3) \times S O(3)$ in $S O(6)$ is given by

$6 \rightarrow(3,1)+(1,3)$.

This implies that the 36 scalars transform as

$(\mathbf{6}, \mathbf{6}) \rightarrow 4 \times(\mathbf{1}+\mathbf{3}+\mathbf{5})$

under the unbroken $S O(3) \sim[S O(3) \times S O(3) \times S O(3) \times$

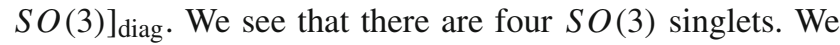
will denote these scalars by $\left(\varphi_{1}, \varphi_{2}, \chi_{1}, \chi_{2}\right)$ as in [20]. In addition, we will also use the explicit parametrization given in [20]. This gives the coset representative

$\mathcal{V}_{M}{ }^{A}=\left(\begin{array}{cc}e^{T} & 0 \\ B e^{T} & e^{-1}\end{array}\right) \otimes \mathbb{I}_{3}$

where the two $2 \times 2$ matrices $e$ and $B$ are defined by

$e=e^{\frac{1}{2}\left(\varphi_{1}+\varphi_{2}\right)}\left(\begin{array}{cc}1 & \chi_{2} \\ 0 & e^{-\varphi_{2}}\end{array}\right), \quad B=\left(\begin{array}{cc}0 & \chi_{1} \\ -\chi_{1} & 0\end{array}\right)$.

Explicitly, the $S O(6,6) / S O(6) \times S O(6)$ coset representative consisting of all $S O(3)$ singlet scalars is given by

$\mathcal{V}_{M}{ }^{A}$

$$
=\left(\begin{array}{cccc}
e^{\frac{1}{2}\left(\varphi_{1}+\varphi_{2}\right)} & 0 & 0 & 0 \\
e^{\frac{1}{2}\left(\varphi_{1}+\varphi_{2}\right)} \chi_{2} & e^{\frac{1}{2}\left(\varphi_{1}-\varphi_{2}\right)} & 0 & 0 \\
e^{\frac{1}{2}\left(\varphi_{1}+\varphi_{2}\right)} \chi_{1} \chi_{2} & e^{\frac{1}{2}\left(\varphi_{1}-\varphi_{2}\right)} \chi_{1} & e^{-\frac{1}{2}\left(\varphi_{1}+\varphi_{2}\right)} & -e^{\frac{1}{2}\left(\varphi_{2}-\varphi_{1}\right)} \chi_{2} \\
-e^{\frac{1}{2}\left(\varphi_{1}+\varphi_{2}\right)} \chi_{1} & 0 & 0 & e^{\frac{1}{2}\left(\varphi_{2}-\varphi_{1}\right)}
\end{array}\right)
$$

$\otimes \mathbb{I}_{3}$.

It should also be noted that there are two scalars which are singlet under $S O(3) \times S O(3) \subset[S O(3) \times S O(3)]_{\text {diag }} \times$ $[S O(3) \times S O(3)]_{\text {diag }}$ as can be seen by taking the tensor product of the representation 6 in (24) giving rise to two singlets $(\mathbf{1}, \mathbf{1})$ of $S O(3) \times S O(3)$. These two singlets correspond to $\varphi_{1}$ and $\varphi_{2}$. 
Similarly, the $S L(2, \mathbb{R}) / S O(2)$ coset representative will be parametrized by

$\mathcal{V}_{\alpha}=e^{\varphi_{g} / 2}\left(\begin{array}{c}\chi_{g}-i e^{-\varphi_{g}} \\ 1\end{array}\right)$

With all these and the definition $\phi^{i}=\left(\varphi_{g}, \varphi_{1}, \varphi_{2}, \chi_{g}, \chi_{1}, \chi_{2}\right)$, the scalar kinetic terms can be found to be

$$
\begin{aligned}
\mathcal{L}_{\text {kin }}= & -\frac{1}{2} K_{i j} \partial_{\mu} \phi^{i} \partial^{\mu} \phi^{j} \\
= & -\frac{1}{4}\left(\partial_{\mu} \varphi_{g} \partial^{\mu} \varphi_{g}\right. \\
& +3 \partial_{\mu} \varphi_{1} \partial^{\mu} \varphi_{1}+3 \partial_{\mu} \varphi_{2} \partial^{\mu} \varphi_{2}+e^{2 \varphi_{g}} \partial_{\mu} \chi_{g} \partial^{\mu} \chi_{g} \\
& \left.+3 e^{2 \varphi_{1}} \partial_{\mu} \chi_{1} \partial^{\mu} \chi_{1}+3 e^{2 \varphi_{2}} \partial_{\mu} \chi_{2} \partial^{\mu} \chi_{2}\right)
\end{aligned}
$$

where we have defined the scalar metric $K_{i j}$, which will play a role in writing the BPS equations.

The four $S O(3)$ singlet scalars in $S O(6,6) / S O(6) \times$ $S O(6)$ correspond to non-compact generators of $S O(2,2) \subset$ $S O(6,6)$ that commute with the $S O(3)$ symmetry. It is convenient to split indices $M=(A I)$ for $A=1,2,3,4$ and $I=1,2,3$. This implies that the $S O(6,6)$ fundamental representation decomposes as $(\mathbf{4}, \mathbf{3})$ under $S O(2,2) \times S O(3)$. In terms of $(A I)$ indices, the embedding tensor can be written as

$$
f_{\alpha M N P}=f_{\alpha A I B J C K}=\Lambda_{\alpha A B C} \epsilon_{I J K}
$$

with $\Lambda_{\alpha A B C}=\Lambda_{\alpha(A B C)}$. In particular, the quadratic constraints read

$\epsilon^{\alpha \beta} \Lambda_{\alpha A B}{ }^{C} \Lambda_{\beta D E C}=0, \quad \Lambda_{(\alpha A[B}^{C} \Lambda_{\beta) D] E C}=0$.

The $S O(6,6)$ fundamental indices $M, N$ can also be decomposed into $(m, \bar{m}), m, \bar{m}=1,2, \ldots, 6$. In connection with the internal manifold $T^{6} / \mathbb{Z}_{2} \times \mathbb{Z}_{2}$, the index $m$ is used to label the $T^{6}$ coordinates and split into $(a, i)$ such that $a=1,3,5$ and $i=2,4,6$. Similar decomposition is also in use for $\bar{m}=(\bar{a}, \bar{i})$. All together, indices $A, B$ can be written as $A=(1,2,3,4)=(a, i, \bar{a}, \bar{i})$. Indices $I, J=1,2,3$ label the three $T^{2}$ 's inside $T^{6} \sim T^{2} \times T^{2} \times T^{2}$.

The $S O(6,6)$ invariant tensor $\eta_{M N}$ and its inverse are chosen to be

$\eta_{M N}=\eta^{M N}=\left(\begin{array}{cc}0 & \mathbb{I}_{6} \\ \mathbb{I}_{6} & 0\end{array}\right)$.

This leads to some extra projections on the negative and positive eigenvalues of $\eta_{M N}$. For example, in order to compute $M_{M N P Q R S}$ in the scalar potential defined by equation (15), we need to project the second index of $\mathcal{V}_{M}{ }^{A}$ by using the projection matrix
$R=\frac{1}{\sqrt{2}}\left(\begin{array}{cc}-\mathbb{I}_{6} & \mathbb{I}_{6} \\ \mathbb{I}_{6} & \mathbb{I}_{6}\end{array}\right)$

Finally, we will also set the gauge coupling $g=\frac{1}{2}$ as in [20].

\section{RG flows from type IIB non-geometric compactification}

We begin with a non-geometric compactification of type IIB theory on $T^{6} / \mathbb{Z}_{2} \times \mathbb{Z}_{2}$. This involves the fluxes of NS and RR three-form fields $\left(H_{3}, F_{3}\right)$ and non-geometric $(P, Q)$ fluxes. This compactification admits a locally geometric description although it is non-geometric in nature.

From the result of [20], the effective $N=4$ gauged supergravity theory is not unique. In this paper, we will only consider the gauged supergravity admitting the maximally supersymmetric $N=4 \mathrm{AdS}_{4}$ vacuum. In this case, all the gauge and non-geometric fluxes lead to the following components of the embedding tensor:

$f_{-\bar{i} \bar{j} \bar{k}}=\Lambda_{-444}=-\lambda, \quad f_{+\bar{a} \bar{b} \bar{c}}=\Lambda_{+333}=\lambda$,

$f_{-\bar{i} \bar{j} k}=\Lambda_{-244}=-\lambda, \quad f_{+a \bar{b} \bar{c}}=\Lambda_{+133}=\lambda$

for a constant $\lambda$. The first and second lines correspond to $\left(H_{3}, F_{3}\right)$ and $(P, Q)$ fluxes, respectively. As shown in [20], the gauge group arising from this embedding tensor is $I S O(3) \times I S O(3) \sim\left[S O(3) \ltimes T^{3}\right] \times\left[S O(3) \ltimes T^{3}\right]$. This gauge group is embedded in $S O(6,6)$ via the $S O(3,3) \times$ $S O(3,3)$ subgroup.

Using this embedding tensor and the explicit form of the scalar coset representative given in the previous section, we find the scalar potential

$$
\begin{aligned}
V= & \frac{1}{32} e^{\varphi_{1}-3 \varphi_{2}-\varphi_{g}} \lambda^{2}\left[e^{2 \varphi_{1}}-3 e^{2 \varphi_{2}}+6 e^{\varphi_{1}+2 \varphi_{2}+\varphi_{g}}\right. \\
& -18 e^{3 \varphi_{2}+\varphi_{g}}-3 e^{4 \varphi_{2}+2 \varphi_{g}} \\
& -2 e^{2 \varphi_{1}+3 \varphi_{2}+\varphi_{g}}\left(1+3 \chi_{1}^{2}\right)+3 e^{2\left(\varphi_{1}+\varphi_{2}\right)}\left(\chi_{1}-\chi_{2}\right)^{2} \\
& -12 e^{5 \varphi_{2}+\varphi_{g}} \chi_{2}^{2} \\
& +3 e^{6 \varphi_{2}} \chi_{2}^{4}+e^{2 \varphi_{1}+6 \varphi_{2}} \chi_{2}^{4}\left(\chi_{2}-3 \chi_{1}\right)^{2} \\
& +3 e^{2 \varphi_{1}+4 \varphi_{2}} \chi_{2}^{2}\left(\chi_{2}-2 \chi_{1}\right)^{2}-3 e^{2\left(\varphi_{2}+\varphi_{g}\right)} \chi_{g}^{2} \\
& +6 e^{\varphi_{1}+4 \varphi_{2}+\varphi_{g}}\left(1+\chi_{2}^{2}\right)+e^{2\left(\varphi_{1}+\varphi_{g}\right)} \chi_{g}^{2} \\
& +3 e^{2\left(\varphi_{1}+\varphi_{2}+\varphi_{g}\right)}\left(\chi_{1}-\chi_{2}\right)^{2} \chi_{g}^{2} \\
& +3 e^{6 \varphi_{2}+2 \varphi_{g}} \chi_{2}^{2}\left(-1+\chi_{2} \chi_{g}\right)^{2} \\
& +3 e^{2\left(\varphi_{1}+2 \varphi_{2}+\varphi_{g}\right)}\left(\chi_{1}-2 \chi_{1} \chi_{2} \chi_{g}+\chi_{2}^{2} \chi_{g}\right)^{2} \\
& \left.+e^{2\left(\varphi_{1}+3 \varphi_{2}+\varphi_{g}\right)}\left[1+\chi_{2}^{3} \chi_{g}-3 \chi_{1} \chi_{2}\left(-1+\chi_{2} \chi_{g}\right)\right]^{2}\right] .
\end{aligned}
$$


Table 1 Scalar masses at the $N=4$ supersymmetric $\mathrm{AdS}_{4}$ critical point with $S O(3) \times S O(3)$ symmetry and the corresponding dimensions of the dual operators

\begin{tabular}{lcl}
\hline Scalar fields & $m^{2} L^{2}$ & $\Delta$ \\
\hline$\varphi_{g}, \chi_{g}$ & -2 & 1,2 \\
$\varphi_{1}, \varphi_{2}$ & 4 & 4 \\
$\chi_{1}, \chi_{2}$ & 0 & 3 \\
\hline
\end{tabular}

This potential admits a trivial critical point at which all scalars vanish. The cosmological constant is given by

$V_{0}=-\frac{3}{8} \lambda^{2}$

At this critical point, we find the scalar masses as in Table 1. In the table, we also give the corresponding dimensions of the dual operators. The $\mathrm{AdS}_{4}$ radius is given by $L=\frac{2 \sqrt{2}}{\lambda}$. Note that we have used different convention for scalar masses from that used in [20]. The masses given in Table 1 are obtained by multiplying the masses given in [20] by 3 .

This $\mathrm{AdS}_{4}$ critical point preserves $N=4$ supersymmetry as can be checked from the $A_{1}^{i j}$ tensor. It should also be emphasized here that this critical point has $S O(3) \times$ $S O$ (3) symmetry which is the maximal compact subgroup of $I S O(3) \times I S O(3)$ gauge group.

To set up the BPS equations for finding supersymmetric RG flow solutions, we first give the metric ansatz

$\mathrm{d} s^{2}=e^{2 A(r)} \mathrm{d} x_{1,2}^{2}+\mathrm{d} r^{2}$

where $\mathrm{d} x_{1,2}^{2}$ is the flat Minkowski metric in three dimensions.

We will use the Majorana representation for gamma matrices with all $\gamma^{\mu}$ real and $\gamma_{5}$ purely imaginary. This choice implies that $\epsilon_{i}$ is a complex conjugate of $\epsilon^{i}$. All scalar fields will be functions of only the radial coordinate $r$. To solve the BPS conditions coming from setting $\delta \chi^{i}=0$ and $\delta \lambda_{a}^{i}=0$, we need the following projection:

$\gamma_{\hat{r}} \epsilon^{i}=e^{i \Lambda} \epsilon^{i}$

From the $\delta \psi_{\mu i}=0$ conditions for $\mu=0,1,2$, we find

$A^{\prime}= \pm W, \quad e^{i \Lambda}= \pm \frac{\mathcal{W}}{W}$

where $W=|\mathcal{W}|$, and ' denotes the $r$-derivative. These equations are obtained by solving real and imaginary parts of $\delta \psi_{\mu i}=0$ separately; see for more details [12,27]. The superpotential $\mathcal{W}$ is defined by

$\mathcal{W}=\frac{1}{3} \alpha$

where $\alpha$ is the eigenvalue of $A_{1}^{i j}$ corresponding to the unbroken supersymmetry. We will choose a definite sign for the $A^{\prime}$ equation and $e^{i \Lambda}$ such that the $N=4$ critical point identified with the $N=4$ SCFT in the UV corresponds to $r \rightarrow \infty$.

For all scalars non-vanishing, the $N=4$ supersymmetry is broken to $N=1$ corresponding to the Killing spinor $\epsilon^{1}$. The superpotential for this unbroken $N=1$ supersymmetry is given by

$$
\begin{aligned}
\mathcal{W}= & \frac{1}{4 \sqrt{2}} e^{\frac{1}{2}\left(\varphi_{1}-3 \varphi_{2}-\varphi_{g}\right)}\left[e ^ { \varphi _ { 2 } } \left[e ^ { \varphi _ { 2 } + \varphi _ { g } } \left(-e^{\varphi_{1}+\varphi_{2}} \lambda\right.\right.\right. \\
& \left.-3 \lambda\left(i+e^{\varphi_{1}} \chi_{1}\right)\left(i+e^{\varphi_{2}} \chi_{2}\right)\right) \\
& -e^{\varphi_{1}} \lambda\left(i+e^{\varphi_{2}} \chi_{2}\right)^{3}\left(i+e^{\varphi_{g}} \chi_{g}\right) \\
& \left.\left.+3 \lambda\left(i+e^{\varphi_{1}} \chi_{1}\right)\left(i+e^{\varphi_{2}} \chi_{2}\right)^{2}\left(i+e^{\varphi_{g}} \chi_{g}\right)\right]\right]
\end{aligned}
$$

from which we find

$$
\begin{aligned}
W= & \frac{1}{8 \sqrt{2}} \lambda e^{\frac{1}{2}\left(\varphi_{1}-3 \varphi_{2}-\varphi_{g}\right)}\left[\left[\left(-3 e^{\varphi_{2}}\left(-e^{\varphi_{1}}+2 e^{\varphi_{2}}\right.\right.\right.\right. \\
& \left.+e^{2 \varphi_{2}+\varphi_{g}}\right) \chi_{2}-e^{\varphi_{1}+3 \varphi_{2}} \chi_{2}^{3} \\
& +e^{\varphi_{g}}\left(e^{\varphi_{1}}-3 e^{\varphi_{2}}\right) \chi_{g}+3 e^{2 \varphi_{2}+\varphi_{g}} \\
& \times\left(-e^{\varphi_{1}}+e^{\varphi_{2}}\right) \chi_{2}^{2} \chi_{g}+3 e^{\varphi_{1}+\varphi_{2}} \chi_{1}(-1 \\
& \left.\left.-e^{\varphi_{2}+\varphi_{g}}+e^{2 \varphi_{2}} \chi_{2}^{2}+2 e^{\varphi_{2}+\varphi_{g}} \chi_{2} \chi_{g}\right)\right)^{2} \\
& +\left[e^{\varphi_{1}}\left(-1+3 e^{2 \varphi_{2}} \chi_{2}^{2}\right)\right. \\
& -e^{\varphi_{1}+\varphi_{2}+\varphi_{g}} \chi_{2}\left(-3+e^{2 \varphi_{2}} \chi_{2}^{2}\right) \chi_{g} \\
& +e^{\varphi_{2}}\left(3+3 e^{\varphi_{2}+\varphi_{g}}-e^{\varphi_{1}+2 \varphi_{2}+\varphi_{g}}\right. \\
& -3 e^{2 \varphi_{2}} \chi_{2}^{2}-6 e^{\varphi_{2}+\varphi_{g}} \chi_{2} \chi_{g} \\
& +3 e^{\varphi_{1}} \chi_{1}\left(-e^{\varphi_{2}}\left(2+e^{\varphi_{2}+\varphi_{g}}\right) \chi_{2}\right. \\
& \left.\left.\left.\left.\left.-e^{\varphi_{g}} \chi_{g}+e^{2 \varphi_{2}+\varphi_{g}} \chi_{2}^{2} \chi_{g}\right)\right)\right]^{2}\right]\right]^{\frac{1}{2}} .
\end{aligned}
$$

The scalar potential can be written in terms of $W$ as

$V=-2 K^{i j} \frac{\partial W}{\partial \phi^{i}} \frac{\partial W}{\partial \phi^{j}}-3 W^{2}$,

and, as usual, the BPS equations from $\delta \chi^{i}=0$ and $\delta \lambda_{a}^{i}=0$ can be written as

$\phi^{i^{\prime}}=2 K^{i j} \frac{\partial W}{\partial \phi^{j}}$.

$K^{i j}$ is the inverse of the scalar kinetic metric defined in (30). The explicit form of these equations is rather complicated and will not be given here. However, they can be found in the appendix.

It is also straightforward to check that these BPS equations solve the second order field equations. Furthermore, there exist a number of interesting subtruncations keeping some subsets of these $S O$ (3) singlets. We will firstly discuss these truncations and consider the full $S O(3)$ singlet sector at the end of this section. 


\subsection{RG flows with $N=4$ supersymmetry}

We begin with RG flow solutions preserving $N=4$ supersymmetry to $N=4$ non-conformal field theories in the IR. The analysis of BPS conditions $\delta \psi_{\mu i}=0, \delta \chi^{i}=0$ and $\delta \lambda_{a}^{i}=0$ shows that there are two possibilities in order to preserve $N=4$ supersymmetry. The first one is to truncate out $\varphi_{1,2}$ and $\chi_{1,2}$. The second possibility is to keep only the three dilatons $\varphi_{g}$ and $\varphi_{1,2}$ by setting $\chi_{g}=\chi_{1,2}=0$.

\subsection{1 $N=4 R G$ flows by relevant deformations}

From Table 1, we see that $\left(\varphi_{g}, \chi_{g}\right)$ correspond to relevant deformations by operators of dimensions 1 or 2 . The BPS equations admit a consistent truncation to these two scalars. By setting $\varphi_{1,2}=\chi_{1,2}=0$, we obtain a set of simple BPS equations

$\varphi_{g}^{\prime}=-\frac{\lambda e^{-\frac{\varphi_{g}}{2}}}{2 \sqrt{2}} \frac{\left(e^{2 \varphi_{g}}+e^{2 \varphi_{g}} \chi_{g}^{2}-1\right)}{\sqrt{\left(1+e^{\varphi_{g}}\right)^{2}+e^{2 \varphi_{g}} \chi_{g}^{2}}}$,

$\chi_{g}^{\prime}=-\frac{\lambda e^{-\frac{\varphi_{g}}{2}}}{\sqrt{2}} \frac{\chi_{g}}{\sqrt{\left(1+e^{\varphi_{g}}\right)^{2}+e^{2 \varphi_{g}} \chi_{g}^{2}}}$,

$A^{\prime}=\frac{\lambda e^{-\frac{\varphi_{g}}{2}}}{4 \sqrt{2}} \sqrt{\left(1+e^{\varphi_{g}}\right)^{2}+e^{2 \varphi_{g}} \chi_{g}^{2}}$.

Since $\left(\varphi_{g}, \chi_{g}\right)$ are scalars in $S L(2, \mathbb{R}) / S O(2)$, they are $S O(6,6)$ singlets and hence $S O(3) \times S O(3)$ invariant. All solutions to these equations then preserve the full $S O(3) \times$ $S O$ (3) symmetry. Moreover, equations $\delta \lambda_{a}^{i}=0$ are identically satisfied, and it can be checked that $N=4$ supersymmetry is unbroken since equations $\delta \chi^{i}=0$ and $\delta \psi_{\mu i}=0$ hold for all $\epsilon^{i}$ satisfying the $\gamma_{\hat{r}}$ projector (39). We should clarify here the convention on the number of supersymmetry. In four dimensions, the $\gamma_{\hat{r}}$ projector reduce the number of supercharges from 16 to 8 . The latter corresponds to $N=4$ supersymmetry in three dimensions. On the other hand, the $\mathrm{AdS}_{4}$ vacuum preserves all 16 supercharges corresponding to $N=4$ superconformal symmetry in three dimensions containing $8+8=16$ supercharges.

We begin with an even simpler solution with $\chi_{g}=0$ which, from the above equations, is clearly a consistent truncation. In this case, we end up with the BPS equations

$$
\begin{aligned}
\varphi_{g}^{\prime} & =-\frac{\lambda}{2 \sqrt{2}} e^{-\frac{\varphi_{g}}{2}}\left(e^{\varphi_{g}}-1\right), \\
A^{\prime} & =\frac{\lambda}{4 \sqrt{2}} e^{-\frac{\varphi_{g}}{2}}\left(1+e^{\varphi_{g}}\right) .
\end{aligned}
$$

The solution to these equations is easily found to be

$$
\varphi_{g}=\ln \left[e^{\frac{r \lambda}{2 \sqrt{2}}+C}-1\right]-\ln \left[e^{\frac{r \lambda}{2 \sqrt{2}}+C}+1\right]
$$

$$
A=\ln \left[e^{\frac{r \lambda}{2 \sqrt{2}}+C}-1\right]-\frac{r \lambda}{2 \sqrt{2}}
$$

with $C$ being an integration constant. The additive integration constant for $A$ has been neglected since it can be absorbed by scaling $\mathrm{d} x_{1,2}^{2}$ coordinates. In addition, the constant $C$ can also be removed by shifting the $r$ coordinate.

At large $r$, we find, as expected for dual operators of dimensions $\Delta=1,2$,

$\varphi_{g} \sim e^{-\frac{\lambda r}{2 \sqrt{2}}} \sim e^{-\frac{r}{L}}$

The solution is singular as $r \rightarrow-\frac{2 \sqrt{2} C}{\lambda}$ since $\varphi_{g} \rightarrow-\infty$. Near this singularity, we find

$\varphi_{g} \sim A \sim \ln \left[r+\frac{2 \sqrt{2} C}{\lambda}\right]$

The scalar potential is bounded above with $V \rightarrow-\infty$. Therefore, the singularity of this solution is physical by the criterion given in [34]. The solution then describes an RG flow from the dual $N=4$ SCFT to an $N=4$ non-conformal field theory with unbroken $S O(3) \times S O(3)$ symmetry. The metric in the IR is given by

$\mathrm{d} s^{2}=(\lambda r+2 \sqrt{2} C)^{2} \mathrm{~d} x_{1,2}^{2}+\mathrm{d} r^{2}$

where we have absorbed some constants to $d x_{1,2}^{2}$ coordinates.

We then consider possible flows solution with $\chi_{g} \neq 0$. By introducing a new variable $\rho$ via

$\frac{\mathrm{d} \rho}{\mathrm{d} r}=\frac{\chi_{g}}{\sqrt{1-C \chi_{g}}+\sqrt{1-\chi_{g}\left(2 C+\chi_{g}\right)}}$

we find the following solution to Eqs. (46)-(48):

$$
\begin{aligned}
\varphi_{g}= & -\frac{1}{2} \ln \left[1-2 C \chi_{g}-\chi_{g}^{2}\right], \\
A= & -\ln \chi_{g}+\frac{1}{4} \ln \left[1-2 C \chi_{g}-\chi_{g}^{2}\right] \\
& +\frac{1}{2} \ln \left[1-C \chi_{g}+\sqrt{1-2 C \chi_{g}-\chi_{g}^{2}}\right], \\
\rho \lambda[1 & \left.-\chi_{g}\left(2 C+\chi_{g}\right)\right]^{\frac{3}{4}} \\
= & 4(2)^{\frac{1}{4}}\left(C+\chi_{g}-\sqrt{1+C^{2}}\right) \\
& \times\left[\frac{1+C^{2}+\sqrt{1+C^{2}}\left(C+\chi_{g}\right)}{1+C^{2}}\right]^{\frac{3}{4}} \\
& \times{ }_{2} F_{1}\left(\frac{1}{4}, \frac{3}{4}, \frac{5}{4}, \frac{\chi_{g}+\sqrt{1+C^{2}}-C}{2 \sqrt{1+C^{2}}}\right)
\end{aligned}
$$

where ${ }_{2} F_{1}$ is the hypergeometric function.

The solution interpolates between the $N=4 \mathrm{AdS}_{4}$ vacuum as $r \rightarrow \infty$ and a singular geometry at a finite value of $r$. 
There are two possibilities for the IR singularities. The first one is given by

$$
\begin{aligned}
& \chi_{g} \sim \chi_{0}, \quad \varphi_{g} \sim-2 \ln \left[\frac{\sqrt{2} r \lambda\left(1+\chi_{0}^{2}\right)-4 \chi_{0} C}{8 \chi_{0}}\right], \\
& A \sim \frac{\chi_{0}}{\sqrt{1+\chi_{0}}} \ln \left[\sqrt{2} r \lambda\left(1+\chi_{0}^{2}\right)-4 \chi_{0} C\right]
\end{aligned}
$$

where $\chi_{0}$ is a constant. In this case, we have $\varphi_{g} \rightarrow \infty$ and $\chi_{g} \rightarrow$ constant as $\sqrt{2} \lambda r\left(1+\chi_{0}^{2}\right) \rightarrow 4 \chi_{0} C$. It should be noted here that the constant $C$ in these equations is not the same as in the full solution given in (57)-(59).

Another possibility is given by

$\varphi_{g} \sim 2 \ln (\sqrt{2} \lambda r+4 C), \quad \chi_{g} \sim \frac{\tilde{C}}{4 C+\sqrt{2} \lambda r}$,

$$
A \sim \ln (\sqrt{2} \lambda r+4 C) .
$$

In this case, as $\sqrt{2} r \lambda \rightarrow-4 C$, we have $\varphi_{g} \rightarrow-\infty$ and $\chi_{g} \rightarrow \pm \infty$ depending on the sign of the constant $\tilde{C}$. Both of these singularities lead to $V \rightarrow-\infty$, so they are physical.

\subsection{2 $N=4 R G$ flows by relevant and irrelevant deformations}

We now consider RG flows with $N=4$ supersymmetry with $\chi_{g}=\chi_{1,2}=0$. Recall that $\varphi_{1}$ and $\varphi_{2}$ are $S O(3) \times$ $S O$ (3) singlets, we still have solutions with $S O(3) \times S O(3)$ unbroken along the flows. It should also be noted that the truncation $\chi_{1,2}=0$ is consistent only for $\chi_{g}=0$. This implies that $N=4$ supersymmetry does not allow turning on the operators dual to $\chi_{g}$ and $\varphi_{1,2}$ simultaneously. It would be interesting to see the implication of this in the dual $N=4$ SCFT.

In this case, the BPS equations reduce to

$$
\begin{aligned}
\varphi_{g}^{\prime}= & \frac{\lambda}{4 \sqrt{2}} e^{\frac{1}{2}\left(\varphi_{1}-3 \varphi_{2}-\varphi_{g}\right)} \\
& \times\left(3 e^{\varphi_{2}}-e^{\varphi_{1}}-3 e^{2 \varphi_{2}+\varphi_{g}}+e^{\varphi_{1}+3 \varphi_{2}+\varphi_{g}}\right), \\
\varphi_{1}^{\prime}= & \frac{\lambda}{4 \sqrt{2}}\left(e^{\varphi_{1}}-e^{\varphi_{2}}-e^{2 \varphi_{2}+\varphi_{g}}+e^{\varphi_{1}+3 \varphi_{2}+\varphi_{g}}\right), \\
\varphi_{2}^{\prime}= & \frac{\lambda}{4 \sqrt{2}}\left(e^{\varphi_{2}}-e^{\varphi_{1}}-e^{2 \varphi_{2}+\varphi_{g}}+e^{\varphi_{1}+3 \varphi_{2}+\varphi_{g}}\right), \\
A^{\prime}= & -\frac{\lambda}{8 \sqrt{2}} e^{\frac{1}{2}\left(\varphi_{1}-3 \varphi_{2}-\varphi_{g}\right)} \\
& \left.\times e^{\varphi_{1}}-3 e^{\varphi_{2}}-3 e^{2 \varphi_{2}+\varphi_{g}}+e^{\varphi_{1}+3 \varphi_{2}+\varphi_{g}}\right) .
\end{aligned}
$$

These equations can be analytically solved by introducing new variables

$\tilde{\varphi}_{1}=\varphi_{1}-\varphi_{2}, \quad \tilde{\varphi}_{2}=\varphi_{1}+\varphi_{2}$ in terms of which the BPS equations become

$\tilde{\varphi}_{1}^{\prime}=\frac{\lambda}{2 \sqrt{2}} e^{\frac{1}{2}\left(\tilde{\varphi}_{1}+\varphi_{g}\right)}\left(e^{\tilde{\varphi}_{1}}-1\right)$,
$\tilde{\varphi}_{2}^{\prime}=\frac{\lambda}{2 \sqrt{2}} e^{\frac{1}{2}\left(\tilde{\varphi}_{2}-\varphi_{g}\right)}\left(e^{\tilde{\varphi}_{2}}-1\right)$,
$\varphi_{g}^{\prime}=\frac{\lambda}{4 \sqrt{2}} e^{-\frac{\varphi_{g}}{2}}\left(3 e^{\frac{\tilde{\varphi}_{2}}{2}}-e^{\frac{3}{2} \tilde{\varphi}_{2}}-3 e^{\frac{1}{2} \tilde{\varphi}_{1}+\varphi_{g}}+e^{\frac{3}{2} \tilde{\varphi}_{1}+\varphi_{g}}\right)$,

$A^{\prime}=-\frac{\lambda}{8 \sqrt{2}} e^{-\frac{\varphi_{g}}{2}}\left(e^{\frac{3}{2} \tilde{\varphi}_{1}+\varphi_{g}}+e^{\frac{3}{2} \tilde{\varphi}_{2}}-3 e^{\frac{\tilde{\varphi}_{2}}{2}}-3 e^{\frac{1}{2} \tilde{\varphi}_{1}+\varphi_{g}}\right)$.

By combining all of these equations, we find that

$$
\begin{aligned}
& \frac{\mathrm{d} A}{\mathrm{~d} \tilde{\varphi}_{1}}-\frac{1}{2} \frac{\mathrm{d} \varphi_{g}}{\mathrm{~d} \tilde{\varphi}_{1}}=\frac{3-e^{\tilde{\varphi}_{1}}}{2\left(e^{\tilde{\varphi}_{1}}-1\right)}, \\
& \frac{\mathrm{d} A}{\mathrm{~d} \tilde{\varphi}_{2}}+\frac{1}{2} \frac{\mathrm{d} \varphi_{g}}{\mathrm{~d} \tilde{\varphi}_{2}}=\frac{3-e^{\tilde{\varphi}_{2}}}{2\left(e^{\tilde{\varphi}_{2}}-1\right)}
\end{aligned}
$$

which can be solved by the following solution:

$$
\begin{aligned}
\varphi_{g} & =\frac{3}{2}\left(\tilde{\varphi}_{1}-\tilde{\varphi}_{2}\right)-\ln \left(1-e^{\tilde{\varphi}_{1}}\right)+\ln \left(1-e^{\tilde{\varphi}_{2}}\right), \\
A & =\frac{\varphi_{g}}{2}-\frac{3}{2} \tilde{\varphi}_{1}+\ln \left(1-e^{\tilde{\varphi}_{1}}\right) .
\end{aligned}
$$

In this solution, we have fixed the integration constant for $\varphi_{g}$ to zero since at the $\mathrm{AdS}_{4}$ critical point $\varphi_{g}=\tilde{\varphi}_{1}=\tilde{\varphi}_{2}=0$. The integration constant for $A$ is irrelevant.

Combining Eqs. (67) and (68), we obtain after substituting for $\varphi_{g}$

$\frac{\mathrm{d} \tilde{\varphi}_{1}}{\mathrm{~d} \tilde{\varphi}_{2}}=e^{2\left(\tilde{\varphi}_{1}-\tilde{\varphi}_{2}\right)}$

whose solution is given by

$\tilde{\varphi}_{1}=-\frac{1}{2} \ln \left(e^{-2 \tilde{\varphi}_{2}}-C_{1}\right)$.

Near the $\mathrm{AdS}_{4}$ critical point, we have $\tilde{\varphi}_{1} \sim \tilde{\varphi}_{2} \sim 0$, which requires that $C_{1}=0$. This choice leads to $\tilde{\varphi}_{2}=\tilde{\varphi}_{1}$, which implies $\varphi_{2}=0$ and $\varphi_{g}=0$. We see that the flow does not involve $\varphi_{g}$ and is driven purely by an irrelevant operator of dimension 4 dual to $\varphi_{1}$. In this case, the $N=4$ SCFT dual to the $\mathrm{AdS}_{4}$ vacuum is expected to appear in the IR. Note also that equation (69) is consistent for $\varphi_{g}=0$ if and only if $\tilde{\varphi}_{2}=\tilde{\varphi}_{1}$ as being the case here.

Finally, we can solve Eq. (67) for $\tilde{\varphi}_{1}(r)$

$$
\frac{\lambda r}{2 \sqrt{2}}=2 e^{-\frac{\tilde{\varphi}_{1}}{2}}+\ln \left(1-e^{-\frac{\tilde{\varphi}_{1}}{2}}\right)-\ln \left(1+e^{-\frac{\tilde{\varphi}_{1}}{2}}\right)+C .
$$


The solution is singular as $r \rightarrow \frac{2 \sqrt{2} C}{\lambda}$. Near this singularity, the solution becomes

$$
\begin{aligned}
\tilde{\varphi}_{1} & \sim \tilde{\varphi}_{2} \sim-\frac{2}{3} \ln \frac{3}{2}\left[C-\frac{\lambda r}{2 \sqrt{2}}\right], \\
A & \sim-\frac{1}{2} \tilde{\varphi}_{1} \sim \frac{1}{3} \ln \frac{3}{2}\left[C-\frac{\lambda r}{2 \sqrt{2}}\right] .
\end{aligned}
$$

This singularity leads to $V \rightarrow \infty$, so the solution is unphysical.

We end the discussion of this truncation by giving some comments on possible subtruncations. From Eqs. (67) and (68), we easily see that setting $\tilde{\varphi}_{1}=0$ or $\tilde{\varphi}_{2}=0$ is a consistent truncation. This is equivalent to setting $\varphi_{2}= \pm \varphi_{1}$. In this case, the solution is found to be

$$
\begin{aligned}
& \varphi_{g}= \pm \ln \left[\frac{e^{-\varphi_{1}}-C_{1} e^{3 \varphi_{1}}}{2-2 e^{2 \varphi_{1}}}\right], \\
& A=-\frac{7}{2} \varphi_{1}+\frac{1}{2} \ln \left(1-e^{2 \varphi_{1}}\right)+\frac{1}{2} \ln \left(1-C_{1} e^{4 \varphi_{1}}\right), \\
& \frac{\lambda \rho}{4 \sqrt{2}}=e^{-\varphi_{1}}+\frac{1}{2} \ln \left(1-e^{-\varphi_{1}}\right)-\frac{1}{2} \ln \left(1+e^{-\varphi_{1}}\right)+C
\end{aligned}
$$

where the new radial coordinate $\rho$ is defined by $\mathrm{d} \rho=$ $e^{-\frac{\varphi_{g}}{2}} \mathrm{~d} r$.

We see that in this case $\varphi_{g}$ is non-trivial along the flow. In order to make the solution approach the $\mathrm{AdS}_{4}$ critical point with $\varphi_{g} \sim \varphi_{1} \sim 0$, we need to choose $C_{1}=1$. This gives

$\varphi_{g}= \pm \ln \cosh \varphi_{1}$

The solution is singular for $\rho \rightarrow \frac{4 \sqrt{2} C}{3 \lambda}$ with $\varphi_{1} \rightarrow \infty$. In this limit, we find

$$
\begin{aligned}
\varphi_{g} & \sim \pm \varphi_{1} \quad \varphi_{1} \sim-\ln \left[C-\frac{3 \lambda \rho}{4 \sqrt{2}}\right], \\
A & \sim \frac{1}{2} \ln \left[C-\frac{3 \lambda \rho}{4 \sqrt{2}}\right] .
\end{aligned}
$$

Both of these singularities lead to $V \rightarrow \infty$, so they are also unphysical.

\subsection{RG flows with $N=1$ supersymmetry}

We now consider a class of RG flow solutions preserving $N=1$ supersymmetry and breaking the $S O(3) \times S O(3)$ to its diagonal subgroup. This is achieved by turning on the marginal deformations corresponding to $\chi_{1}$ and $\chi_{2}$ to the solutions. As in the $N=4$ case, there is a consistent subtruncation to only irrelevant and marginal deformations with only $\varphi_{1}$ and $\chi_{1}$ non-vanishing. We will consider this case first and then look for the most general solutions with all six $S O$ (3) singlet scalars non-vanishing. It should be noted that the truncation with only $\varphi_{2}$ and $\chi_{2}$ non-vanishing is not consistent. This is also an interesting feature to look for in the dual field theory.

\subsection{1 $N=1 R G$ flows by marginal and irrelevant deformations}

By setting $\varphi_{g}=\chi_{g}=\varphi_{2}=\chi_{2}=0$ in the BPS equations, we obtain

$$
\begin{aligned}
& \varphi_{1}^{\prime}=-\frac{\lambda}{2 \sqrt{2}} e^{\frac{\varphi_{1}}{2}} \frac{\left(3-4 e^{\varphi_{1}}+e^{2 \varphi_{1}}+9 \chi_{1}^{2} e^{2 \varphi_{1}}\right)}{\sqrt{\left(e^{\varphi_{1}}-3\right)^{2}+9 \chi_{1}^{2} e^{2 \varphi_{1}}}}, \\
& \chi_{1}^{\prime}=-\frac{3 \lambda}{2 \sqrt{2}} \frac{\chi_{1} e^{\frac{\varphi_{1}}{2}}}{\sqrt{\left(e^{\varphi_{1}}-3\right)^{2}+9 \chi_{1}^{2} e^{2 \varphi_{1}}}}, \\
& A^{\prime}=\frac{\lambda}{4 \sqrt{2}} e^{\frac{\varphi_{1}}{2}} \sqrt{\left(e^{\varphi_{1}}-3\right)^{2}+9 \chi_{1}^{2} e^{2 \varphi_{1}}} .
\end{aligned}
$$

We are not able to analytically solve these equations in full generality, so we will look for numerical solutions in this case.

Note that further truncation to only $\varphi_{1}$ gives rise to the following BPS equations:

$$
\varphi_{1}^{\prime}=\frac{\lambda}{2 \sqrt{2}} e^{\frac{\varphi_{1}}{2}}\left(e^{\varphi_{1}}-1\right) \text { and } A^{\prime}=-\frac{\lambda}{4 \sqrt{2}} e^{\frac{\varphi_{1}}{2}}\left(e^{\varphi_{1}}-3\right) \text {, }
$$

with the solution

$$
\begin{aligned}
& A=-\frac{3}{2} \varphi_{1}+\ln \left(1-e^{\varphi_{1}}\right), \\
& \frac{\lambda r}{2 \sqrt{2}}=2 e^{-\frac{\varphi_{1}}{2}}+\ln \left(1-e^{-\frac{\varphi_{1}}{2}}\right)-\ln \left(1+e^{-\frac{\varphi_{1}}{2}}\right) .
\end{aligned}
$$

This is nothing but the solution of the previous section for $\tilde{\varphi}_{2}=\tilde{\varphi}_{1}$. Therefore, we will not further discuss this solution.

For non-vanishing $\chi_{1}$, we need to find the solutions numerically. An example of these solutions is given in Fig. 1.

The asymptotic behavior of this solution can be determined from the BPS equations at large $\varphi_{1}$ as follows:

$$
\begin{aligned}
\chi_{1} & \sim \chi_{0}, \quad \varphi_{1} \sim-\frac{2}{3} \ln \left(r \lambda \sqrt{2+18 \chi_{0}^{2}}-4 C_{1}\right), \\
A & \sim \frac{1}{3} \ln \left(r \lambda \sqrt{2+18 \chi_{0}^{2}}-4 C_{1}\right)
\end{aligned}
$$

where $\chi_{0}$ is a constant. This singularity leads to $V \rightarrow \infty$, implying that it is unphysical. We have in addition checked this by a numerical analysis which consistently shows a diverging scalar potential near the singularity. 


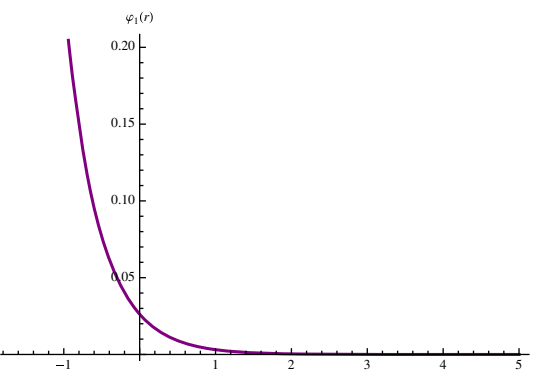

(a) Solution for $\varphi_{1}$

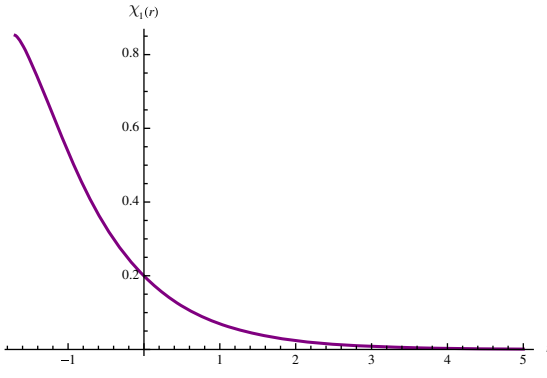

(b) Solution for $\chi_{1}$

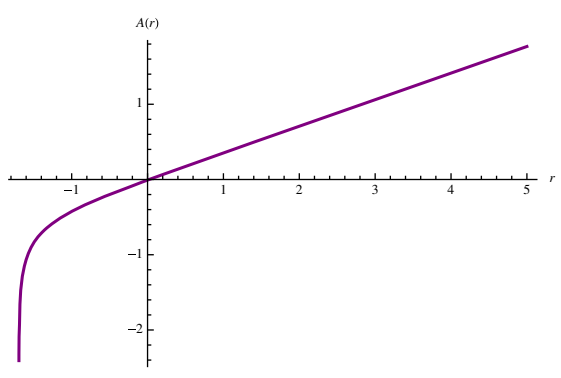

(c) Solution for $A$

Fig. 1 An $N=1$ RG flow with marginal and irrelevant deformations from type IIB compactification

\subsection{2 $N=1 R G$ flows by relevant, marginal and irrelevant deformations}

After considering various consistent truncations, we end this section by considering $N=1 \mathrm{RG}$ flow solutions with all six $S O$ (3) singlet scalars turned on. The resulting RG flows will be driven by all types of possible deformations namely marginal, irrelevant and relevant. In this case, we need to use a numerical analysis due to the complexity of the full set of BPS equations given in the Appendix. Similar to the analysis of [9], there could be many possible IR singularities due to the competition between various deformations both by operators and vacuum expectation values (vev) present in the UV $N=4$ SCFT. Some examples of these solutions are given in Fig. 2. In the figure, we have given solutions for three different values of the flux parameter $\lambda$ for comparison.

From Fig. 2, we see a singularity in the IR end of the flow while near $r \rightarrow \infty$ the flow approaches the UV $N=4$ $\mathrm{AdS}_{4}$. The numerical analysis shows that the singularity is of a bad type according to the criterion of [34] since it leads to $V \rightarrow \infty$.

\section{Supersymmetric Janus solutions}

In this section, we look at another type of solutions with an $\mathrm{AdS}_{3}$-sliced domain wall ansatz, obtained by replacing the flat metric $\mathrm{d} x_{1,2}^{2}$ by an $\mathrm{AdS}_{3}$ metric of radius $\ell$,

$\mathrm{d} s^{2}=e^{2 A(r)}\left(e^{\frac{2 \xi}{\ell}} \mathrm{d} x_{1,1}^{2}+\mathrm{d} \xi^{2}\right)+\mathrm{d} r^{2}$.

The solution, called Janus solution, describes a conformal interface of co-dimension one within the SCFT dual to the $\mathrm{AdS}_{4}$ critical point. This solution breaks the threedimensional conformal symmetry $\operatorname{SO}(2,3)$ to that on the $(1+1)$-dimensional interface $S O(2,2)$.

In this case, the resulting BPS equations will get modified compared to the RG flow case. First of all, the analysis of $\delta \psi_{\mu}^{i}=0$ equations requires an additional $\gamma_{\hat{\xi}}$ projection $\gamma_{\hat{\xi}} \epsilon_{i}=i \kappa e^{i \Lambda} \epsilon^{i}$

while the $\gamma_{\hat{r}}$ projector in $\delta \chi^{i}=0$ and $\delta \lambda_{a}^{i}=0$ equations is still given by Eq. (39) but with the phase $e^{i \Lambda}$ modified to

$e^{i \Lambda}=\frac{\mathcal{W}}{A^{\prime}+\frac{i \kappa}{\ell} e^{-A}}$.

Furthermore, the integrability conditions for $\delta \psi_{\hat{0}, \hat{1}}^{i}=0$ equations lead to

$A^{\prime 2}+\frac{1}{\ell^{2}} e^{-2 A}=W^{2}$

As expected, these two equations reduce to $A^{\prime}= \pm W$ and $e^{i \Lambda}=\frac{\mathcal{W}}{A^{\prime}}= \pm \frac{\mathcal{W}}{W}$ in the limit $\ell \rightarrow \infty$

The constant $\kappa$, with $\kappa^{2}=1$, imposes the chirality condition on the Killing spinors corresponding to the unbroken supersymmetry on the $(1+1)$-dimensional interface. The detailed analysis of these equations can be found for example in [27]. Unlike the RG flow case, the Killing spinors depend on both $r$ and $\xi$ coordinates; see for more details [26].

We have seen that the analysis of RG flow solutions with all six $S O$ (3) singlet scalars turned on involves a very complicated set of BPS equations. Since the BPS equations for supersymmetric Janus solutions are usually more complicated than those of the RG flows, we will not perform the full analysis with all $S O(3)$ singlet scalars but truncate the BPS equations to two consistent truncations, with $\left(\varphi_{g}, \chi_{g}\right)$ and $\left(\varphi_{1}, \chi_{1}\right)$ non-vanishing. As in other cases studied in $[14,26,27]$, truncations to only dilatons or scalars without the axions or pseudoscalars are not consistent with the Janus BPS equations, or equivalently Janus solutions require nontrivial pseudoscalars.

\section{1 $N=4$ Janus solution}

We first consider the Janus solution with only the dilaton and axion in the gravity multiplet non-vanishing. In this case, the BPS conditions $\delta \lambda_{a}^{i}=0$ are automatically satisfied by 


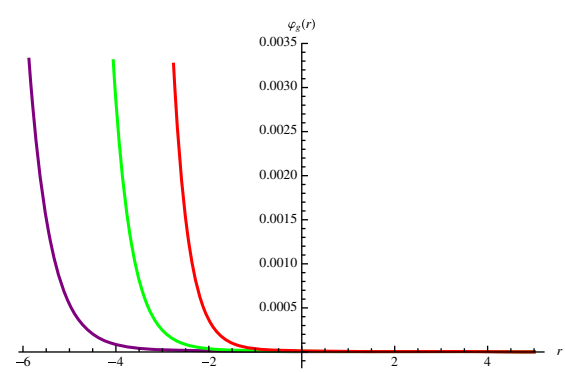

(a) Solution for $\varphi_{g}$

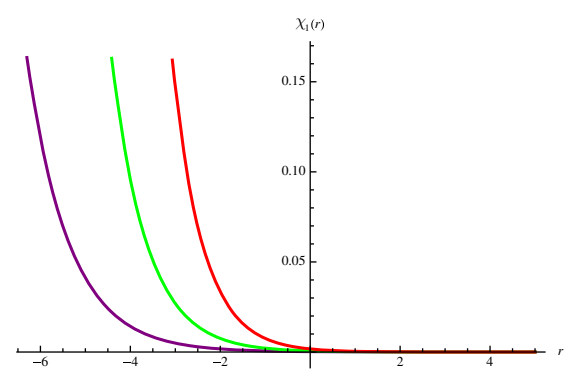

(d) Solution for $\chi_{1}$

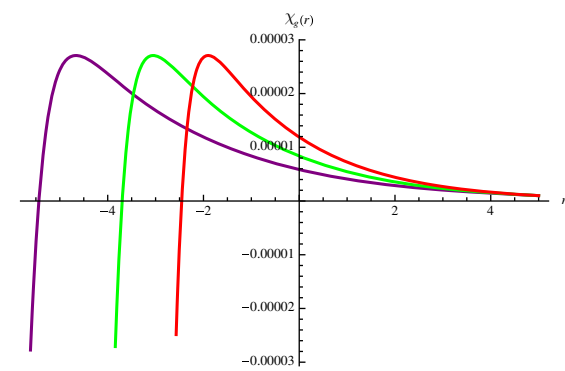

(b) Solution for $\chi_{g}$

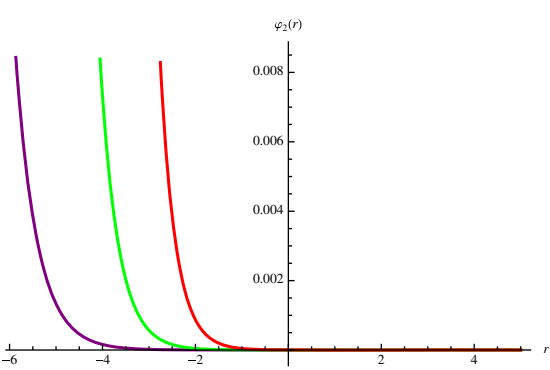

(e) Solution for $\varphi_{2}$

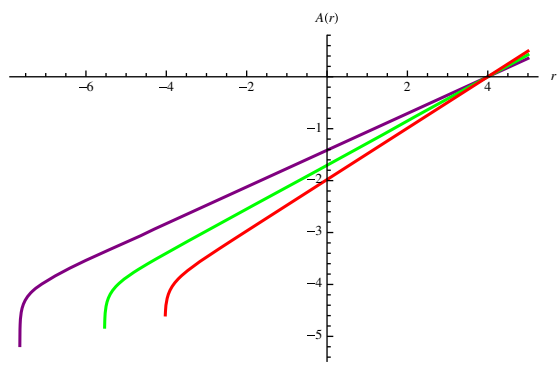

(g) Solution for $A$

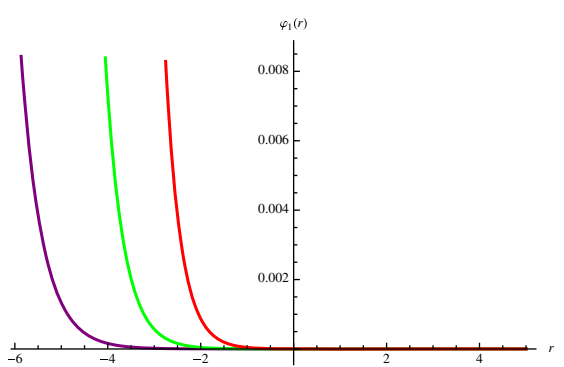

(c) Solution for $\varphi_{1}$

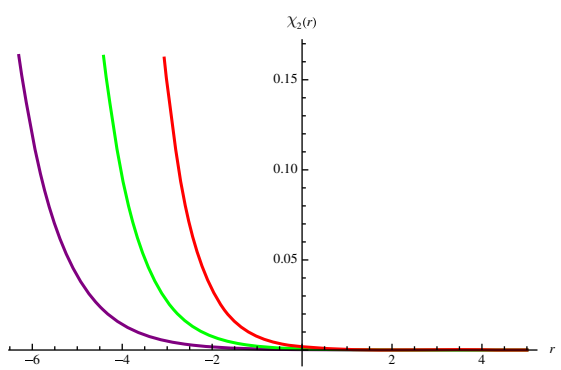

(f) Solution for $\chi_{2}$

Fig. $2 N=1$ RG flow solutions from type IIB compactification with all $S O(3)$ singlet scalars and $\lambda=1($ purple $), \lambda=1.2($ green $)$ and $\lambda=1.4$ (red)

setting $\varphi_{1,2}=\chi_{1,2}=0$. By using the phase (90) in $\delta \chi^{i}=0$ equations and separating real and imaginary parts, we obtain the following BPS equations:

$$
\begin{aligned}
\varphi_{g}^{\prime} & =-4 \frac{A^{\prime}}{W} \frac{\partial W}{\partial \varphi_{g}}-4 \kappa e^{-\varphi_{g}} \frac{e^{-A}}{\ell W} \frac{\partial W}{\partial \chi_{g}} \\
& =\frac{-2 \ell A^{\prime}\left(e^{2 \varphi_{g}}-1+2 \chi_{g}^{2} e^{2 \varphi_{g}}\right)-4 \kappa e^{\varphi_{g}-A} \chi_{g}}{\ell\left[\left(1+e^{\varphi_{g}}\right)^{2}+\chi_{g}^{2} e^{2 \varphi_{g}}\right]}, \\
\chi_{g}^{\prime} & =-4 \frac{A^{\prime}}{W} e^{-2 \varphi_{g}} \frac{\partial W}{\partial \chi_{g}}+4 \kappa e^{-\varphi_{g}} \frac{e^{-A}}{\ell W} \frac{\partial W}{\partial \varphi_{g}} \\
& =\frac{2 \kappa e^{-A-\varphi_{g}}\left(e^{2 \varphi_{g}}-1+\chi_{g}^{2} e^{2 \varphi_{g}}\right)-4 \ell \chi_{g} A^{\prime}}{\ell\left[\left(1+e^{\varphi_{g}}\right)^{2}+\chi_{g}^{2} e^{2 \varphi_{g}}\right]}, \\
0 & =A^{\prime 2}+\frac{e^{-2 A}}{\ell^{2}}-\frac{\lambda^{2}}{32} e^{-\varphi_{g}}\left[\left(1+e^{\varphi_{g}}\right)^{2}+\chi_{g}^{2} e^{2 \varphi_{g}}\right]
\end{aligned}
$$

where we have also included the gravitini equations from (91). In terms of the superpotential

$W=\frac{\lambda}{4 \sqrt{2}} e^{-\frac{\varphi_{g}}{2}} \sqrt{\left(1+e^{\varphi_{g}}\right)^{2}+\chi_{g}^{2} e^{2 \varphi_{g}}}$,

these equations take a similar form as in the other fourdimensional Janus solutions in $[14,26,27]$. These equations solve all the BPS conditions for any $\epsilon^{i}, i=1,2,3,4$. Therefore, any solutions to these equations will preserve $N=4$ supersymmetry. We solve these equations numerically with an example of the solutions given in Fig. 3.

From Fig. 3, we see that the solution interpolates between $N=4 \mathrm{AdS}_{4}$ vacua at both $r \rightarrow \pm \infty$. This solution is then interpreted as a $(1+1)$-dimensional conformal interface within the $N=4$ SCFT. The interface preserves $N=(4,0)$ supersymmetry on the interface due to the sign choice $\kappa=1$, 


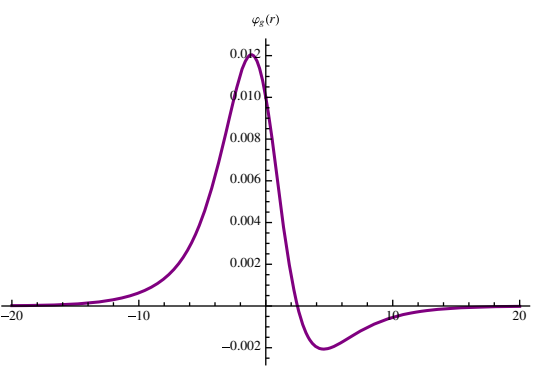

(a) Solution for $\varphi_{g}$

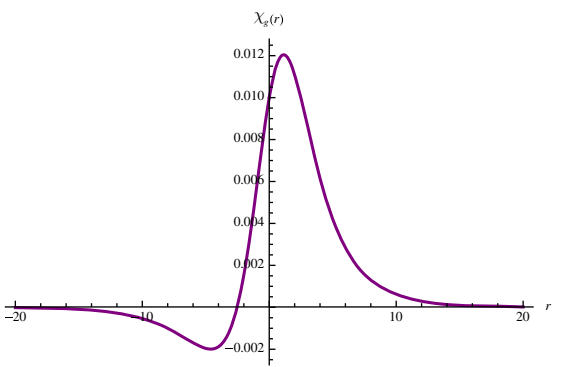

(b) Solution for $\chi_{g}$

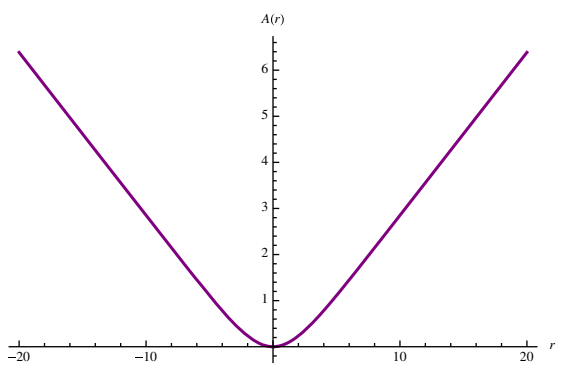

(c) Solution for $A$

Fig. $3 N=4$ Janus solution from type IIB compactification within a truncation to $\varphi_{g}$ and $\chi_{g}$ with $\kappa=\lambda=1$ and $\ell=2 \sqrt{2}$

and $S O(3) \times S O(3)$ symmetry remains unbroken throughout the solution.

\section{$4.2 N=1$ Janus solution}

The truncation keeping only $\varphi_{1}$ and $\chi_{1}$ is still consistent in the case of Janus BPS equations. In contrast to the previous truncation, any solutions to these equations will break $N=4$ supersymmetry to $N=1$ and preserve only $S O$ (3) diagonal subgroup of the full $S O(3) \times S O(3)$ symmetry of the $N=4$ $\mathrm{AdS}_{4}$ vacuum.

The real superpotential for this truncation is given by

$W=\frac{\lambda}{4 \sqrt{2}} e^{\frac{\varphi_{1}}{2}} \sqrt{\left(e^{\varphi_{1}}-3\right)^{2}+9 \chi_{1}^{2} e^{2 \varphi_{1}}}$

in terms of which the BPS equations can be written as

$$
\begin{aligned}
\varphi_{1}^{\prime} & =-\frac{4}{3} \frac{A^{\prime}}{W} \frac{\partial W}{\partial \varphi_{1}}-\frac{4}{3} \kappa e^{-\varphi_{1}} \frac{e^{-A}}{\ell W} \frac{\partial W}{\partial \chi_{1}} \\
& =\frac{2 \ell A^{\prime}\left(4 e^{2 \varphi_{1}}-3-9 \chi_{1}^{2} e^{2 \varphi_{1}}-e^{2 \varphi_{1}}\right)-12 \kappa e^{\varphi_{1}-A} \chi_{1}}{\ell\left[\left(e^{\varphi_{1}}-3\right)^{2}+9 \chi_{1}^{2} e^{2 \varphi_{1}}\right]} \\
\chi_{1}^{\prime} & =-\frac{4}{3} \frac{A^{\prime}}{W} e^{-2 \varphi_{1}} \frac{\partial W}{\partial \chi_{1}}+\frac{4}{3} \kappa e^{-\varphi_{1}} \frac{e^{-A}}{\ell W} \frac{\partial W}{\partial \varphi_{1}} \\
& =\frac{2 \kappa e^{-A-\varphi_{1}}\left(3-4 e^{\varphi_{1}}+e^{2 \varphi_{1}}+9 \chi_{1}^{2} e^{2 \varphi_{1}}\right)-12 \ell \chi_{1} A^{\prime}}{\ell\left[\left(e^{\varphi_{1}}-3\right)^{2}+9 \chi_{1}^{2} e^{2 \varphi_{1}}\right]} \\
0 & =A^{\prime 2}+\frac{e^{-2 A}}{\ell^{2}}-\frac{\lambda^{2}}{32} e^{\varphi_{1}}\left[\left(e^{\varphi_{1}}-3\right)^{2}+9 \chi_{1}^{2} e^{2 \varphi_{1}}\right]
\end{aligned}
$$

Unlike the previous case, an intensive numerical search has not found any solutions interpolating between $\mathrm{AdS}_{4}$ vacua in the limits $r \rightarrow \pm \infty$. All of the solutions found here are singular Janus in the sense that they connect singular domain walls at two finite values of the radial coordinate. We give an example of these solutions in Fig. 4.

This solution could be interpreted as a conformal interface between two $N=1$ non-conformal phases of the dual $N=4$ SCFT. However, the singularities are of the bad type.
An uplift to type IIB theory would be needed in order to decide whether the solution is physically acceptable in the ten-dimensional context.

\section{RG flows from type IIA geometric compactification}

We now carry out a similar analysis for a geometric compactification of type IIA theory. The procedure is essentially the same, so we will omit unnecessary details. In this case, the compactification only involves gauge $\left(H_{3}, F_{0}, F_{2}, F_{4}, F_{6}\right)$ and geometric $(\omega)$ fluxes. However, the fluxes are more complicated and lead to many components of the embedding tensor compared to the type IIB case

$H_{i j k} \sim f_{-\bar{a} \bar{b} \bar{c}}=\Lambda_{-333}=\frac{\sqrt{6}}{3} \lambda$,

$F_{\text {aibjck }} \sim f_{+\bar{a} \bar{b} \bar{c}}=\Lambda_{+333}=-\frac{3 \sqrt{10}}{2} \lambda$,

$F_{a i b j} \sim f_{+\bar{a} \bar{b} \bar{k}}=\Lambda_{+334}=\frac{\sqrt{6}}{2} \lambda$,

$F_{a i} \sim f_{+\bar{a} \bar{j} \bar{k}}=\Lambda_{+344}=\frac{\sqrt{10}}{6} \lambda$,

$F_{0} \sim f_{+\bar{i} \bar{j} \bar{k}}=\Lambda_{+444}=\frac{5 \sqrt{6}}{6} \lambda$,

$H_{a b k} \sim f_{+\bar{a} \bar{b} k}=\Lambda_{+233}=\frac{\sqrt{6}}{3} \lambda$,

$\omega_{i j}{ }^{c} \sim f_{-\bar{a} \bar{b} \bar{k}}=\Lambda_{-334}=\frac{\sqrt{10}}{3} \lambda$,

$\omega_{k a}{ }^{j}=\omega_{b k}{ }^{i}=\omega_{b c}{ }^{a} \sim f_{+\bar{a} \bar{j} k}=f_{+\bar{i} \bar{b} k}$

$=f_{+a \bar{b} \bar{c}}=\Lambda_{+234}=\Lambda_{+133}=\sqrt{10} \lambda$.

In the above equations, we have also given the form field corresponding to each flux component.

The resulting gauged $N=4$ supergravity has a nonsemisimple group ISO(3) $\ltimes U(1)^{6}$ and admits the minimal $N=1 \mathrm{AdS}_{4}$ vacuum at which the gauge group is broken down to $S O$ (3) compact subgroup. The corresponding superpotential for the unbroken $N=1$ supersymmetry is given by 


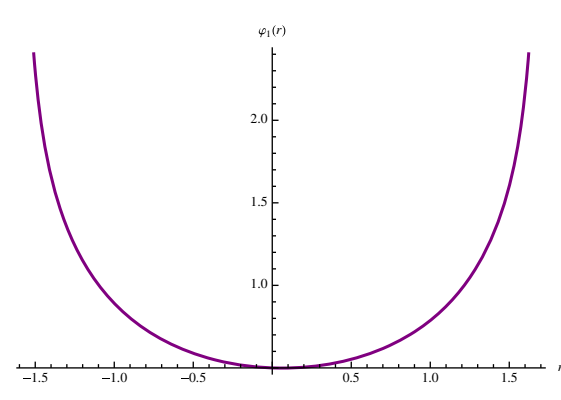

(a) Solution for $\varphi_{1}$

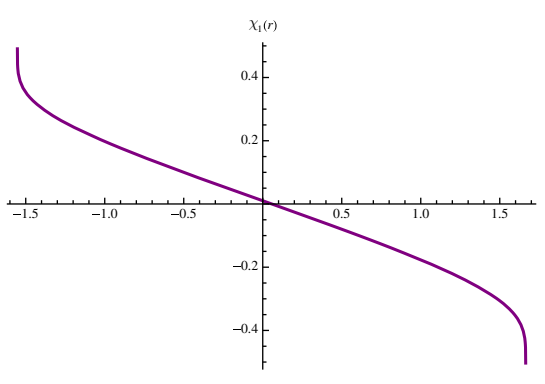

(b) Solution for $\chi_{1}$

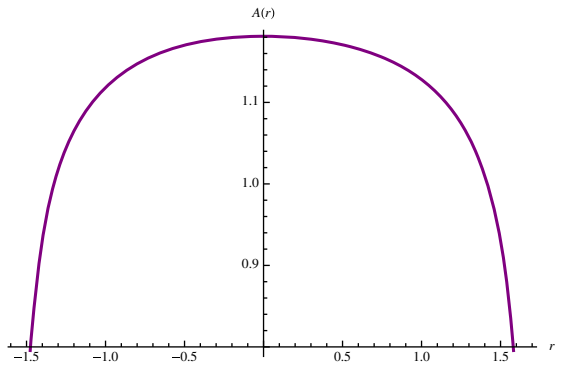

(c) Solution for $A$

Fig. $4 N=1$ Janus solution from type IIB compactification within a truncation to $\varphi_{1}$ and $\chi_{1}$ with $\kappa=\lambda=\ell=1$

$$
\begin{aligned}
\mathcal{W}= & \frac{\lambda}{24} e^{\frac{1}{2}\left(\varphi_{1}-3 \varphi_{2}+\varphi_{g}\right)}\left[2 e^{\varphi_{1}+2 \varphi_{2}-\varphi_{g}}\right. \\
& \times\left[3 \sqrt{5} i+e^{2 \varphi_{2}}\left(\sqrt{3}+3 \sqrt{5} \chi_{2}\right)\right]\left(i+\chi_{g} e^{\varphi_{g}}\right) \\
& -5 \sqrt{3} e^{\varphi_{1}}\left(i+e^{\varphi_{2}} \chi_{2}\right)^{3}-3 \sqrt{5} e^{\varphi_{1}+\varphi_{2}}\left(i+e^{\varphi_{2}} \chi_{2}\right)^{2} \\
& -9 \sqrt{3} i e^{\varphi_{1}+2 \varphi_{2}} \\
& +18 \sqrt{5} e^{2 \varphi_{2}}\left(i+e^{\varphi_{1}} \chi_{1}\right)\left(i+e^{\varphi_{2}} \chi_{2}\right) \\
& +6 \sqrt{3} i e^{3 \varphi_{2}}+9 \sqrt{5} e^{\varphi_{1}+3 \varphi_{2}} \\
& \left.+6 \sqrt{3} e^{\varphi_{1}+3 \varphi_{2}} \chi_{1}-9 \sqrt{3} \chi_{2} e^{\varphi_{1}+3 \varphi_{2}}\right]
\end{aligned}
$$

The scalar potential can be written in terms of $W=|\mathcal{W}|$ as

$V=-\frac{1}{2} K^{i j} \frac{\partial W}{\partial \phi^{i}} \frac{\partial W}{\partial \phi^{j}}-\frac{3}{4} W^{2}$.

Its explicit form is given in the appendix.

When all scalars vanish, there is an $N=1 \mathrm{AdS}_{4}$ vacuum with the cosmological constant

$V_{0}=-\lambda^{2}$

The six scalars have squared masses as follows:

$m^{2} L^{2}: 0,-2,4 \pm \sqrt{6}, \frac{1}{3}(47 \pm \sqrt{159})$.

All of these values are in agreement with [20] after changing to our convention including a factor of 3 .

As in the type IIB case, the BPS equations obtained from supersymmetry variations can be written as

$A^{\prime}=W, \quad \varphi^{i^{\prime}}=K^{i j} \frac{\partial W}{\partial \phi^{j}}$.

However, the resulting equations are much more complicated than those from type IIB compactification. We will then not give them in this paper. Furthermore, we have not found any consistent subtruncation within this set of equations. In the following, we will only give examples of holographic RG flows from the $N=1 \mathrm{SCFT}$ dual to the above $\mathrm{AdS}_{4}$ critical point to non-conformal $N=1$ field theories in the IR. These numerical solutions are shown in Fig. 5 with three different values of the flux parameter $\lambda$ as in the IIB case.

As in the IIB case, we have numerically analyzed the scalar potential near the singularity and found that it leads to $V \rightarrow$ $\infty$, which implies the singularity is unphysical.

\section{Conclusions and discussions}

We have found many supersymmetric RG flows and examples of Janus solutions in $N=4$ gauged supergravities obtained from flux compactifications of type II string theories. These solutions describe supersymmetric deformations and conformal interfaces within the dual $N=4$ and $N=1$ SCFTs in three dimensions. Many of the flow solutions have been obtained analytically which should be useful for further investigation.

In type IIB non-geometric compactification, the gauged supergravity has $I S O(3) \times I S O(3)$ gauge group and admits an $N=4 \mathrm{AdS}_{4}$ vacuum dual to an $N=4$ SCFT with global symmetry $S O(3) \times S O(3)$. We have found two classes of supersymmetric RG flows. The first one preserves $N=4$ supersymmetry, and the global $S O(3) \times S O(3)$ symmetry is unbroken. This type of solutions can be obtained by turning on only the dilaton and axion in the gravity multiplet dual to relevant operators of dimensions $\Delta=1,2$. In this case, the flows are accordingly driven by relevant operators. Another possibility for preserving $N=4$ supersymmetry is to truncate out all axions or pseudoscalars. The resulting $\mathrm{RG}$ flows are driven by relevant and irrelevant operators of dimensions $\Delta=1,2$ and $\Delta=4$, respectively. When the axions in the vector multplets, corresponding to marginal deformations, are turned on, the flows break $N=4$ supersymmetry to $N=1$ and break $S O(3) \times S O(3)$ symmetry to their $S O$ (3) diagonal subgroup. We have given numerically the flows driven by marginal and irrelevant operators and the most general deformations in the presence of all types of deformations, relevant, marginal and irrelevant. It has been pointed out in [20] that the vacuum structure of type IIB com- 


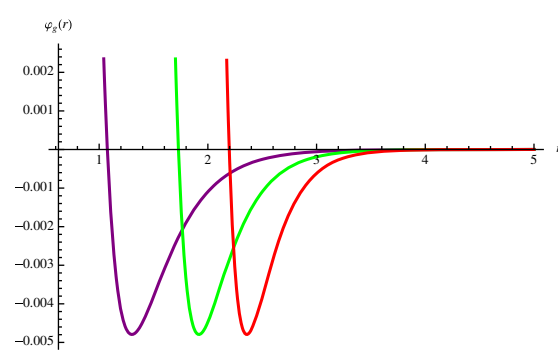

(a) Solution for $\varphi_{g}$

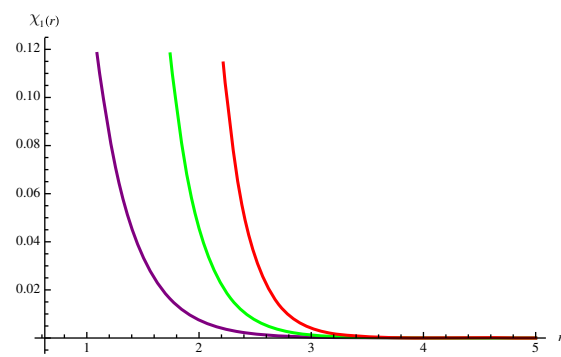

(d) Solution for $\chi_{1}$

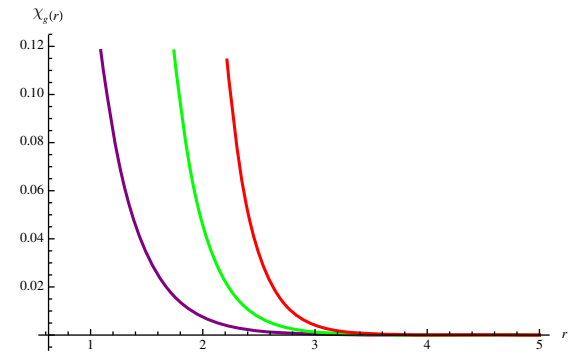

(b) Solution for $\chi_{g}$

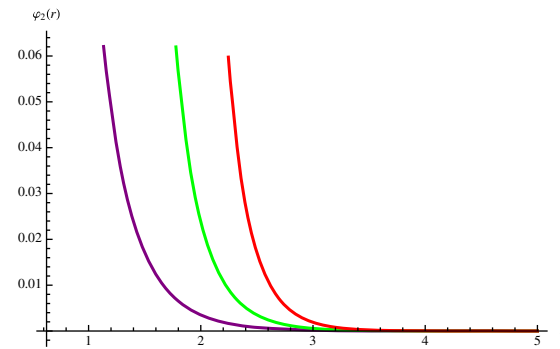

(e) Solution for $\varphi_{2}$

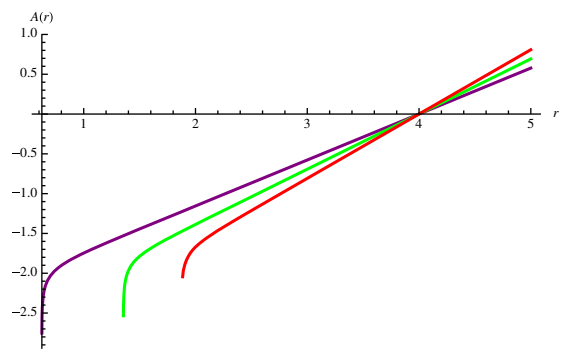

(g) Solution for $A$

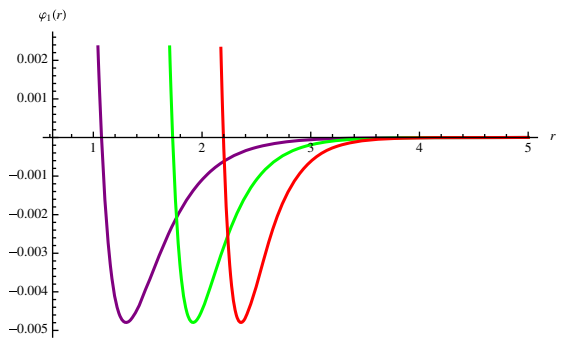

(c) Solution for $\varphi_{1}$

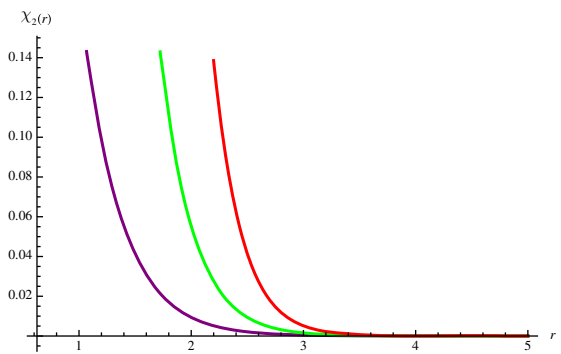

(f) Solution for $\chi_{2}$

Fig. $5 N=1$ RG flow solutions from type IIA compactification with $\lambda=1$ (purple), $\lambda=1.2($ green $)$ and $\lambda=1.4$ (red)

pactification is very rich. The solutions found in this paper show that the number of supersymmetric deformations of these vacua is also enormous.

Within this type IIB compactification, we have also given Janus solutions preserving $N=4$ and $N=1$ supersymmetry. These correspond to $(1+1)$-dimensional conformal interfaces preserving $S O(3) \times S O(3)$ and $S O(3)$ symmetry. For the $N=4$ solution, we have given a numerical solution interpolating between $\mathrm{AdS}_{4}$ vacua on the two sides of the interface, called regular Janus. This solution gives a holographic dual of a conformal interface in $N=4$ SCFT. For the $N=1$ case, we have not found this type of solutions but the singular Janus, interpolating between $N=1$ nonconformal phases of the dual $N=4$ SCFT. The situation is very similar to the $N=1$ Janus solutions studied in [14]. It would be interesting to have a definite conclusion about the existence of regular Janus solutions in these two $N=4$ gauged supergravities.
In this non-geometric compactification, it is useful to give some comments about the holographic interpretation of the results. Due to its non-geometric nature, the stringy origin of the $N=4$ gauged supergravity is presently not well understood. This makes the meaning of the resulting solutions in terms of RG flows in the dual SCFT unclear. However, working in four-dimensional gauged supergravity has an obvious advantage in the sense that the whole formulation of $N=4$ gauged supergravity is virtually unchanged for all gaugings from both geometric and non-geometric compactifications. Therefore, the approach used here can be carried out for all other gaugings regardless of their higher-dimensional origins. On the other hand, the full interpretation of the results in higher-dimensional contexts calls for further study. Hopefully, the results presented here could be useful along this line of investigations.

We have also carried out the same analysis in a geometric compactification of type IIA theory resulting in $N=4$ gauged supergravity with $I S O(3) \ltimes U(1)^{6}$. The gauged 
supergravity admits an $N=1 \mathrm{AdS}_{4}$ vacuum dual to an $N=$ 1 SCFT in three dimensions. Due to the lack of further consistent subtruncation, we have numerically found examples of holographic RG flows to $N=1$ non-conformal field theories. Similar to the solutions in the type IIB case, the flows are driven by relevant, marginal and irrelevant operators in a more complicated manner. It should be pointed out that the massless scalars dual to marginal deformations considered in this paper are not the Goldstone bosons corresponding to the symmetry breaking $I S O(3) \times I S O(3) \rightarrow S O(3) \times S O(3)$ and $I S O(3) \ltimes U(1)^{6} \rightarrow S O(3)$. The Goldstone bosons transform non-trivially under the residual symmetry groups $S O(3) \times S O(3)$ and $S O(3)$ while the massless scalars considered in the solutions are singlets. Therefore, they are truly marginal deformations in the $N=1$ and $N=4$ SCFTs. Note also that, in the type IIB case, these marginal deformations break $N=4$ supersymmetry in consistent with the fact that all maximally supersymmetric $\mathrm{AdS}_{4}$ vacua of $N=4$ gauged supergravity have no moduli preserving $N=4$ supersymmetry [35].

There are many possibilities for further investigations. First of all, it would be interesting to identify the $N=1$ and $N=4$ SCFTs dual to the $N=1$ and $N=4 \mathrm{AdS}_{4}$ vacua. This should allow us to identify the dual operators driving the RG flows obtained holographically in this paper. It could be interesting to look for more general Janus solutions in type IIB compactification with more scalars turned on and also look for similar solutions in type IIA compactification. Another direction would be to uplift the solutions found here to ten dimensions. This could be used to identify the $g_{00}$ component of the ten-dimensional metric and checked whether the unphysical singularities by the criterion of [34] are physical by the criterion of [36]. Finally, it would be of particular interest to further explore type IIB compactification with more general fluxes than those considered in [20]. This could enlarge the solution space of both $\mathrm{AdS}_{4}$ vacua and their deformations including possible flow solutions between two $\mathrm{AdS}_{4}$ vacua. We leave these issues for future work.

Acknowledgements This work is supported by The Thailand Research Fund (TRF) under Grant RSA5980037.

Open Access This article is distributed under the terms of the Creative Commons Attribution 4.0 International License (http://creativecomm ons.org/licenses/by/4.0/), which permits unrestricted use, distribution, and reproduction in any medium, provided you give appropriate credit to the original author(s) and the source, provide a link to the Creative Commons license, and indicate if changes were made.

Funded by SCOAP $^{3}$.

\section{Appendix A: Useful formulas}

In this appendix, we collect all of the conventions about 't Hooft symbols, the scalar potential coming from type IIA geometric compactification and complicated BPS equations arising from type IIB non-geometric compactification with all $S O(3)$ singlet scalars non-vanishing.

\section{A.1: 't Hooft symbols}

To convert an $S O(6)$ vector index $m$ to a pair of antisymmetric $S U(4)$ indices $[i j]$, we use the following 't Hooft symbols:

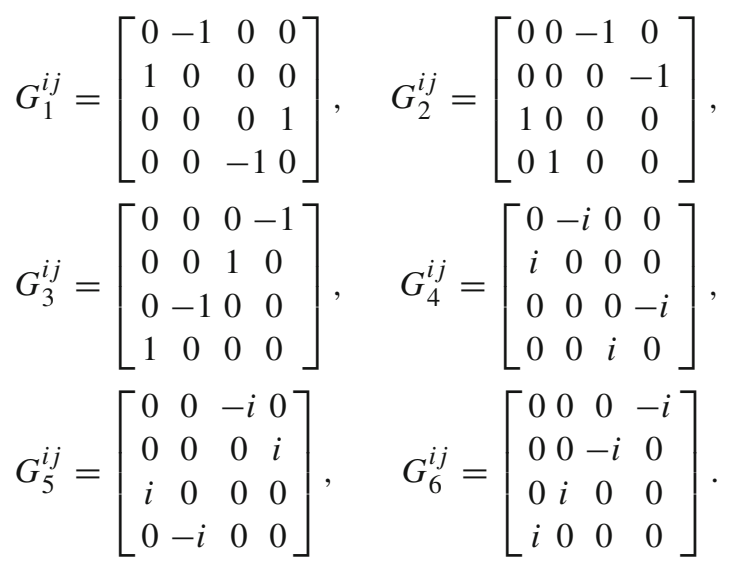

These matrices satisfy the relation

$G_{m i j}=-\frac{1}{2} \epsilon_{i j k l} G_{m}^{k l}=-\left(G_{m}^{i j}\right)^{*}$.

\section{A.2: BPS equations for type IIB compactification}

In this section, we give the full BPS equations for the nongeometric compactification of type IIB theory. These equations are given by

$$
\begin{aligned}
\varphi_{g}^{\prime}= & -\frac{1}{32 W} e^{\varphi_{1}-3 \varphi_{2}-\varphi_{g}} \lambda^{2}\left[-e^{2 \varphi_{1}}-9 e^{2 \varphi_{2}}\right. \\
& +6 e^{\varphi_{1}+\varphi_{2}}+e^{2\left(\varphi_{1}+3 \varphi_{2}+\varphi_{g}\right)} \\
& +9 e^{4 \varphi_{2}+2 \varphi_{g}}-6 e^{\varphi_{1}+5 \varphi_{2}+2 \varphi_{g}} \\
& +6 e^{3 \varphi_{2}+2 \varphi_{g}}\left(2 e^{\varphi_{1}}-3 e^{\varphi_{2}}-e^{2 \varphi_{1}+\varphi_{2}}\right. \\
& \left.+2 e^{\varphi_{1}+2 \varphi_{2}}\right) \chi_{2} \chi_{g}+2 e^{5 \varphi_{2}+2 \varphi_{g}} \\
& \times\left(6 e^{\varphi_{1}}-9 e^{\varphi_{2}}+e^{2 \varphi_{1}+\varphi_{2}}\right) \chi_{2}^{3} \chi_{g}+e^{2\left(\varphi_{1}+\varphi_{g}\right)} \chi_{g}^{2} \\
& +9 e^{2\left(\varphi_{2}+\varphi_{g}\right)} \chi_{g}^{2}-6 e^{\varphi_{1}+\varphi_{2}+2 \varphi_{g}} \chi_{g}^{2} \\
& +3 e^{4 \varphi_{2}}\left(e^{2 \varphi_{1}}+3 e^{2 \varphi_{2}}-2 e^{\varphi_{1}+\varphi_{2}}\right) \chi_{2}^{4}(-1 \\
& \left.+e^{2 \varphi_{g}} \chi_{g}^{2}\right)+e^{2 \varphi_{1}+6 \varphi_{2}} \chi_{2}^{6}\left(-1+e^{2 \varphi_{g}} \chi_{g}^{2}\right) \\
& +3 e^{2 \varphi_{2}} \chi_{2}^{2}\left(-e^{2 \varphi_{1}}-6 e^{2 \varphi_{2}}+4 e^{\varphi_{1}+\varphi_{2}}\right. \\
& +3 e^{4 \varphi_{2}+2 \varphi_{g}}+e^{2 \varphi_{g}}\left(e^{2 \varphi_{1}}+6 e^{2 \varphi_{2}}\right. \\
& \left.\left.-4 e^{\varphi_{1}+\varphi_{2}}\right) \chi_{g}^{2}\right)+9 e^{2\left(\varphi_{1}+\varphi_{2}\right)} \chi_{1}^{2}(1 \\
& \left.+e^{2 \varphi_{2}} \chi_{2}^{2}\right)\left(-1+e^{2\left(\varphi_{2}+\varphi_{g}\right)}-2 e^{2\left(\varphi_{2}+\varphi_{g}\right)} \chi_{2} \chi_{g}\right.
\end{aligned}
$$




$$
\begin{aligned}
& \left.+e^{2 \varphi_{g}} \chi_{g}^{2}+e^{2 \varphi_{2}} \chi_{2}^{2}\left(-1+e^{2 \varphi_{g}} \chi_{g}^{2}\right)\right) \\
& -6 e^{2\left(\varphi_{1}+\varphi_{2}\right)} \chi_{1}\left(-e^{2 \varphi_{g}}\left(-1+e^{2 \varphi_{2}}\right) \chi_{g}\right. \\
& +e^{4 \varphi_{2}+2 \varphi_{g}} \chi_{2}^{2} \chi_{g}-e^{4 \varphi_{2}+2 \varphi_{g}} \chi_{2}^{4} \chi_{g} \\
& -\chi_{2}\left(1+e^{4 \varphi_{2}+2 \varphi_{g}}-e^{2 \varphi_{g}} \chi_{g}^{2}\right) \\
& \left.\left.+2 e^{2 \varphi_{2}} \chi_{2}^{3}\left(-1+e^{2 \varphi_{g}} \chi_{g}^{2}\right)+e^{4 \varphi_{2}} \chi_{2}^{5}\left(-1+e^{2 \varphi_{g}} \chi_{g}^{2}\right)\right)\right],
\end{aligned}
$$

$$
\begin{aligned}
\chi_{g}^{\prime}= & -\frac{1}{16 W} e^{\varphi_{1}-3 \varphi_{2}-\varphi_{g}} \lambda^{2}\left[3 e ^ { 3 \varphi _ { 2 } } \left(2 e^{\varphi_{1}}-3 e^{\varphi_{2}}\right.\right. \\
& \left.-e^{2 \varphi_{1}+\varphi_{2}}+2 e^{\varphi_{1}+2 \varphi_{2}}\right) \chi_{2} \\
& +e^{5 \varphi_{2}}\left(6 e^{\varphi_{1}}-9 e^{\varphi_{2}}+e^{2 \varphi_{1}+\varphi_{2}}\right) \chi_{2}^{3} \\
& +\left(e^{\varphi_{1}}-3 e^{\varphi_{2}}\right)^{2} \chi_{g}+3 e^{2 \varphi_{2}}\left(e^{2 \varphi_{1}}+6 e^{2 \varphi_{2}}\right. \\
& \left.-4 e^{\varphi_{1}+\varphi_{2}}\right) \chi_{2}^{2} \chi_{g}+3 e^{4 \varphi_{2}}\left(e^{2 \varphi_{1}}+3 e^{2 \varphi_{2}}\right. \\
& \left.-2 e^{\varphi_{1}+\varphi_{2}}\right) \chi_{2}^{4} \chi_{g}+e^{2 \varphi_{1}+6 \varphi_{2}} \chi_{2}^{6} \chi_{g} \\
& +9 e^{2\left(\varphi_{1}+\varphi_{2}\right)} \chi_{1}^{2}\left(1+e^{2 \varphi_{2}} \chi_{2}^{2}\right)\left(-e^{2 \varphi_{2}} \chi_{2}+\chi_{g}\right. \\
& \left.+e^{2 \varphi_{2}} \chi_{2}^{2} \chi_{g}\right)-3 e^{2\left(\varphi_{1}+\varphi_{2}\right)} \chi_{1}(1 \\
& -e^{2 \varphi_{2}}+e^{4 \varphi_{2}} \chi_{2}^{2}-e^{4 \varphi_{2}} \chi_{2}^{4}+2 \chi_{2} \chi_{g} \\
& \left.\left.+4 e^{2 \varphi_{2}} \chi_{2}^{3} \chi_{g}+2 e^{4 \varphi_{2}} \chi_{2}^{5} \chi_{g}\right)\right],
\end{aligned}
$$

$$
\begin{aligned}
\varphi_{1}^{\prime}= & -\frac{1}{32 W} e^{\varphi_{1}-3 \varphi_{2}-\varphi_{g}} \lambda^{2}\left[e^{2 \varphi_{1}}+3 e^{2 \varphi_{2}}\right. \\
& -4 e^{\varphi_{1}+\varphi_{2}}-4 e^{\varphi_{1}+2 \varphi_{2}+\varphi_{g}}+6 e^{3 \varphi_{2}+\varphi_{g}} \\
& +e^{2\left(\varphi_{1}+3 \varphi_{2}+\varphi_{g}\right)}+2 e^{2 \varphi_{1}+3 \varphi_{2}+\varphi_{g}} \\
& -4 e^{\varphi_{1}+4 \varphi_{2}+\varphi_{g}}+3 e^{4 \varphi_{2}+2 \varphi_{g}}-4 e^{\varphi_{1}+5 \varphi_{2}+2 \varphi_{g}} \\
& +2 e^{3 \varphi_{2}+2 \varphi_{g}}\left(4 e^{\varphi_{1}}-3 e^{\varphi_{2}}-3 e^{2 \varphi_{1}+\varphi_{2}}\right. \\
& \left.+4 e^{\varphi_{1}+2 \varphi_{2}}\right) \chi_{2} \chi_{g}+2 e^{5 \varphi_{2}+2 \varphi_{g}}\left(4 e^{\varphi_{1}}\right. \\
& \left.-3 e^{\varphi_{2}}+e^{2 \varphi_{1}+\varphi_{2}}\right) \chi_{2}^{3} \chi_{g}+e^{2\left(\varphi_{1}+\varphi_{g}\right)} \chi_{g}^{2} \\
& +3 e^{2\left(\varphi_{2}+\varphi_{g}\right)} \chi_{g}^{2}-4 e^{\varphi_{1}+\varphi_{2}+2 \varphi_{g}} \chi_{g}^{2} \\
& +e^{2 \varphi_{1}+6 \varphi_{2}} \chi_{2}^{6}\left(1+e^{2 \varphi_{g}} \chi_{g}^{2}\right)+e^{2 \varphi_{2}} \chi_{2}^{2}\left(3 e^{2 \varphi_{1}}\right. \\
& +6 e^{2 \varphi_{2}}-8 e^{\varphi_{1}+\varphi_{2}}+6 e^{3 \varphi_{2}+\varphi_{g}} \\
& -6 e^{2 \varphi_{1}+3 \varphi_{2}+\varphi_{g}}+4 e^{\varphi_{1}+4 \varphi_{2}+\varphi_{g}} \\
& +3 e^{4 \varphi_{2}+2 \varphi_{g}}+e^{2 \varphi_{g}}\left(3 e^{2 \varphi_{1}}+6 e^{2 \varphi_{2}}\right. \\
& \left.\left.-8 e^{\varphi_{1}+\varphi_{2}}\right) \chi_{g}^{2}\right)+e^{4 \varphi_{2}} \chi_{2}^{4}\left(3 e^{2 \varphi_{1}}+3 e^{2 \varphi_{2}}\right. \\
& -4 e^{\varphi_{1}+\varphi_{2}}+4 e^{\varphi_{1}+2 \varphi_{2}+\varphi_{g}} \\
& \left.+e^{2 \varphi_{g}}\left(3 e^{2 \varphi_{1}}+3 e^{2 \varphi_{2}}-4 e^{\varphi_{1}+\varphi_{2}}\right) \chi_{g}^{2}\right) \\
& +9 e^{2\left(\varphi_{1}+\varphi_{2}\right)} \chi_{1}^{2}\left(1+e^{2 \varphi_{2}} \chi_{2}^{2}\right)\left(\left(1+e^{\varphi_{2}+\varphi_{g}}\right)^{2}\right. \\
& \left.-2 e^{2\left(\varphi_{2}+\varphi_{g}\right)} \chi_{2} \chi_{g}+e^{2 \varphi_{g}} \chi_{g}^{2}+e^{2 \varphi_{2}} \chi_{2}^{2}\left(1+e^{2 \varphi_{g}} \chi_{g}^{2}\right)\right) \\
& -6 e^{2\left(\varphi_{1}+\varphi_{2}\right)} \chi_{1}\left(-e^{2 \varphi_{g}}(-1\right. \\
& \left.+e^{2 \varphi_{2}}\right) \chi_{g}+e^{4 \varphi_{2}+2 \varphi_{g}} \chi_{2}^{2} \chi_{g} \\
& -e^{4 \varphi_{2}+2 \varphi_{g}} \chi_{2}^{4} \chi_{g}+e^{4 \varphi_{2}} \chi_{2}^{5}\left(1+e^{2 \varphi_{g}} \chi_{g}^{2}\right) \\
& +2 e^{2 \varphi_{2}} \chi_{2}^{3}\left(1+e^{\varphi_{2}+\varphi_{g}}\right. \\
&
\end{aligned}
$$

$$
\begin{aligned}
\varphi_{2}^{\prime}= & -\frac{1}{32 W} e^{\varphi_{1}-3 \varphi_{2}-\varphi_{g}} \lambda^{2}\left[-e^{2 \varphi_{1}}-3 e^{2 \varphi_{2}}\right. \\
& +4 e^{\varphi_{1}+\varphi_{2}}+2 e^{\varphi_{1}+2 \varphi_{2}+\varphi_{g}} \\
& +e^{2\left(\varphi_{1}+3 \varphi_{2}+\varphi_{g}\right)}-2 e^{\varphi_{1}+4 \varphi_{2}+\varphi_{g}} \\
& +3 e^{4 \varphi_{2}+2 \varphi_{g}}-4 e^{\varphi_{1}+5 \varphi_{2}+2 \varphi_{g}}+2 e^{4 \varphi_{2}+2 \varphi_{g}}(-3 \\
& \left.-e^{2 \varphi_{1}}+4 e^{\varphi_{1}+\varphi_{2}}\right) \chi_{2} \chi_{g} \\
& +2 e^{5 \varphi_{2}+2 \varphi_{g}}\left(4 e^{\varphi_{1}}-9 e^{\varphi_{2}}+e^{2 \varphi_{1}+\varphi_{2}}\right) \chi_{2}^{3} \chi_{g} \\
& -e^{2\left(\varphi_{1}+\varphi_{g}\right)} \chi_{g}^{2}-3 e^{2\left(\varphi_{2}+\varphi_{g}\right)} \chi_{g}^{2} \\
& +4 e^{\varphi_{1}+\varphi_{2}+2 \varphi_{g}} \chi_{g}^{2}+e^{2 \varphi_{1}+6 \varphi_{2}} \chi_{2}^{6}\left(1+e^{2 \varphi_{g}} \chi_{g}^{2}\right) \\
& +e^{2 \varphi_{2}} \chi_{2}^{2}\left(-e^{2 \varphi_{1}}+6 e^{2 \varphi_{2}}+12 e^{3 \varphi_{2}+\varphi_{g}}\right. \\
& -4 e^{2 \varphi_{1}+3 \varphi_{2}+\varphi_{g}}+6 e^{\varphi_{1}+4 \varphi_{2}+\varphi_{g}} \\
& \left.+9 e^{4 \varphi_{2}+2 \varphi_{g}}-e^{2 \varphi_{g}}\left(e^{2 \varphi_{1}}-6 e^{2 \varphi_{2}}\right) \chi_{g}^{2}\right) \\
& +e^{4 \varphi_{2}} \chi_{2}^{4}\left(e^{2 \varphi_{1}}+9 e^{2 \varphi_{2}}-4 e^{\varphi_{1}+\varphi_{2}}\right. \\
& +6 e^{\varphi_{1}+2 \varphi_{2}+\varphi_{g}}+e^{2 \varphi_{g}}\left(e^{2 \varphi_{1}}+9 e^{2 \varphi_{2}}\right. \\
& \left.\left.-4 e^{\varphi_{1}+\varphi_{2}}\right) \chi_{g}^{2}\right)-2 e^{2\left(\varphi_{1}+\varphi_{2}\right)} \chi_{1}\left(-e^{2 \varphi_{g}}(1\right. \\
& \left.+e^{2 \varphi_{2}}\right) \chi_{g}+3 e^{4 \varphi_{2}+2 \varphi_{g}} \chi_{2}^{2} \chi_{g}-3 e^{4 \varphi_{2}+2 \varphi_{g}} \chi_{2}^{4} \chi_{g} \\
& +3 e^{4 \varphi_{2}} \chi_{2}^{5}\left(1+e^{2 \varphi_{g}} \chi_{g}^{2}\right) \\
& +2 e^{2 \varphi_{2}} \chi_{2}^{3}\left(1+2 e^{\varphi_{2}+\varphi_{g}}+e^{2 \varphi_{g}} \chi_{g}^{2}\right)-\chi_{2}\left(1+4 e^{3 \varphi_{2}+\varphi_{g}}\right. \\
& \left.\left.+3 e^{4 \varphi_{2}+2 \varphi_{g}}+e^{2 \varphi_{g}} \chi_{g}^{2}\right)\right) \\
& +3 e^{2\left(\varphi_{1}+\varphi_{2}\right)} \chi_{1}^{2}\left(-1+e^{2\left(\varphi_{2}+\varphi_{g}\right)}-2 e^{2\left(\varphi_{2}+\varphi_{g}\right)} \chi_{2} \chi_{g}\right. \\
& -6 e^{4 \varphi_{2}+2 \varphi_{g}} \chi_{2}^{3} \chi_{g}-e^{2 \varphi_{g}} \chi_{g}^{2} \\
& +3 e^{4 \varphi_{2}} \chi_{2}^{4}\left(1+e^{2 \varphi_{g}} \chi_{g}^{2}\right)+e^{2 \varphi_{2}} \chi_{2}^{2}\left(2+4 e^{\varphi_{2}+\varphi_{g}}\right. \\
& \left.\left.\left.+3 e^{2\left(\varphi_{2}+\varphi_{g}\right)}+2 e^{2 \varphi_{g}} \chi_{g}^{2}\right)\right)\right], \\
&
\end{aligned}
$$

$$
\begin{aligned}
\chi_{1}^{\prime}= & \frac{1}{16 W} e^{\varphi_{1}-\varphi_{2}-\varphi_{g}} \lambda^{2}\left[-e^{2 \varphi_{g}}\left(-1+e^{2 \varphi_{2}}\right) \chi_{g}\right. \\
& +e^{4 \varphi_{2}+2 \varphi_{g}} \chi_{2}^{2} \chi_{g}-e^{4 \varphi_{2}+2 \varphi_{g}} \chi_{2}^{4} \chi_{g} \\
& +e^{4 \varphi_{2}} \chi_{2}^{5}\left(1+e^{2 \varphi_{g}} \chi_{g}^{2}\right)+2 e^{2 \varphi_{2}} \chi_{2}^{3} \\
& \times\left(1+e^{\varphi_{2}+\varphi_{g}}+e^{2 \varphi_{g}} \chi_{g}^{2}\right)+\chi_{2}\left(1+2 e^{\varphi_{2}+\varphi_{g}}\right. \\
& \left.-2 e^{3 \varphi_{2}+\varphi_{g}}-e^{4 \varphi_{2}+2 \varphi_{g}}+e^{2 \varphi_{g}} \chi_{g}^{2}\right) \\
& -3 \chi_{1}\left(1+e^{2 \varphi_{2}} \chi_{2}^{2}\right)\left(\left(1+e^{\varphi_{2}+\varphi_{g}}\right)^{2}\right. \\
& -2 e^{2\left(\varphi_{2}+\varphi_{g}\right)} \chi_{2} \chi_{g}+e^{2 \varphi_{g}} \chi_{g}^{2} \\
& \left.\left.+e^{2 \varphi_{2}} \chi_{2}^{2}\left(1+e^{2 \varphi_{g}} \chi_{g}^{2}\right)\right)\right],
\end{aligned}
$$$$
\chi_{2}^{\prime}=-\frac{1}{16 W} e^{\varphi_{1}-3 \varphi_{2}-\varphi_{g}} \lambda^{2}\left[e ^ { \varphi _ { 2 } + 2 \varphi _ { g } } \left(2 e^{\varphi_{1}}-3 e^{\varphi_{2}}\right.\right.
$$$$
\left.-e^{2 \varphi_{1}+\varphi_{2}}+2 e^{\varphi_{1}+2 \varphi_{2}}\right) \chi_{g}
$$$$
+e^{3 \varphi_{2}+2 \varphi_{g}}\left(6 e^{\varphi_{1}}-9 e^{\varphi_{2}}\right.
$$ 


$$
\begin{aligned}
& \left.+e^{2 \varphi_{1}+\varphi_{2}}\right) \chi_{2}^{2} \chi_{g}+e^{2 \varphi_{1}+4 \varphi_{2}} \chi_{2}^{5}\left(1+e^{2 \varphi_{g}} \chi_{g}^{2}\right) \\
& +\chi_{2}\left(e^{2 \varphi_{1}}+6 e^{2 \varphi_{2}}-4 e^{\varphi_{1}+\varphi_{2}}\right. \\
& +6 e^{3 \varphi_{2}+\varphi_{g}}-2 e^{2 \varphi_{1}+3 \varphi_{2}+\varphi_{g}}+2 e^{\varphi_{1}+4 \varphi_{2}+\varphi_{g}} \\
& +3 e^{4 \varphi_{2}+2 \varphi_{g}}+e^{2 \varphi_{g}}\left(e^{2 \varphi_{1}}+6 e^{2 \varphi_{2}}\right. \\
& \left.\left.-4 e^{\varphi_{1}+\varphi_{2}}\right) \chi_{g}^{2}\right)+2 e^{2 \varphi_{2}} \chi_{2}^{3}\left(e^{2 \varphi_{1}}+3 e^{2 \varphi_{2}}\right. \\
& -2 e^{\varphi_{1}+\varphi_{2}}+2 e^{\varphi_{1}+2 \varphi_{2}+\varphi_{g}}+e^{2 \varphi_{g}}\left(e^{2 \varphi_{1}}\right. \\
& \left.\left.+3 e^{2 \varphi_{2}}-2 e^{\varphi_{1}+\varphi_{2}}\right) \chi_{g}^{2}\right)-e^{2 \varphi_{1}} \chi_{1}(1 \\
& +2 e^{\varphi_{2}+\varphi_{g}}-2 e^{3 \varphi_{2}+\varphi_{g}}-e^{4 \varphi_{2}+2 \varphi_{g}} \\
& +2 e^{4 \varphi_{2}+2 \varphi_{g}} \chi_{2} \chi_{g}-4 e^{4 \varphi_{2}+2 \varphi_{g}} \chi_{2}^{3} \chi_{g} \\
& +e^{2 \varphi_{g}} \chi_{g}^{2}+5 e^{4 \varphi_{2}} \chi_{2}^{4}\left(1+e^{2 \varphi_{g}} \chi_{g}^{2}\right) \\
& \left.+6 e^{2 \varphi_{2}} \chi_{2}^{2}\left(1+e^{\varphi_{2}+\varphi_{g}}+e^{2 \varphi_{g}} \chi_{g}^{2}\right)\right) \\
& +3 e^{2\left(\varphi_{1}+\varphi_{2}\right)} \chi_{1}^{2}\left(-e^{2 \varphi_{g}} \chi_{g}-3 e^{2\left(\varphi_{2}+\varphi_{g}\right)} \chi_{2}^{2} \chi_{g}\right. \\
& +2 e^{2 \varphi_{2}} \chi_{2}^{3}\left(1+e^{2 \varphi_{g}} \chi_{g}^{2}\right) \\
& \left.\left.+\chi_{2}\left(2+2 e^{\varphi_{2}+\varphi_{g}}+e^{2\left(\varphi_{2}+\varphi_{g}\right)}+2 e^{2 \varphi_{g}} \chi_{g}^{2}\right)\right)\right]
\end{aligned}
$$

where $W$ is given in (43).

\section{A.3: Scalar potential from type IIA compactification}

The scalar potential obtained from a geometric compactification of type IIA theory is given by

$$
\begin{aligned}
& V= \frac{1}{192} e^{\varphi_{1}-3 \varphi_{2}-\varphi_{g}} \lambda^{2}\left[20 e^{2 \varphi_{1}+4 \varphi_{2}}\right. \\
&+25 e^{2\left(\varphi_{1}+\varphi_{g}\right)}-240 e^{\varphi_{1}+4 \varphi_{2}+\varphi_{g}}-180 e^{4 \varphi_{2}+2 \varphi_{g}} \\
&+5 e^{2\left(\varphi_{1}+\varphi_{2}+\varphi_{g}\right)}\left(1+2 \sqrt{15} \chi_{2}+15 \chi_{2}^{2}\right) \\
&+12 e^{6 \varphi_{2}+2 \varphi_{g}}\left(1+2 \sqrt{15} \chi_{2}+15 \chi_{2}^{2}\right) \\
&+e^{2 \varphi_{1}+6 \varphi_{2}}\left(4+8 \sqrt{15} \chi_{2}+60 \chi_{2}^{2}\right) \\
&+e^{2\left(\varphi_{1}+2 \varphi_{2}+\varphi_{g}\right)}\left[180 \chi_{1}^{2}-12 \chi_{1}(3 \sqrt{15}\right. \\
&\left.+5 \chi_{2}\left(2+\sqrt{15} \chi_{2}\right)-10 \chi_{g}\right) \\
&+3\left(9+4 \sqrt{15} \chi_{2}-4 \sqrt{15} \chi_{g}\right)+5\left[4 \sqrt{15} \chi_{2}^{3}\right. \\
&\left.\left.+15 \chi_{2}^{4}-8 \chi_{2} \chi_{g}+4 \chi_{g}^{2}+\chi_{2}^{2}\left(22-4 \sqrt{15} \chi_{g}\right)\right]\right] \\
&+e^{2\left(\varphi_{1}+3 \varphi_{2}+\varphi_{g}\right)}[135 \\
&-54 \sqrt{15} \chi_{2}+10 \sqrt{15} \chi_{2}^{5} \\
&+25 \chi_{2}^{6}+12 \sqrt{15} \chi_{g}-4 \chi_{2}^{3}\left(3 \sqrt{15}+60 \chi_{1}+20 \chi_{g}\right) \\
&+8 \chi_{2}\left(3 \chi_{1}+\chi_{g}\right)\left(18+3 \sqrt{15} \chi_{1}+\sqrt{15} \chi_{g}\right) \\
&-5 \chi_{2}^{4}\left(-21+12 \sqrt{15} \chi_{1}\right. \\
&\left.+4 \sqrt{15} \chi_{g}\right)+4\left[9 \chi_{1}\left(\sqrt{15}+\chi_{1}\right)\right. \\
&\left.+6 \chi_{1} \chi_{g}+\chi_{g}^{2}\right]+\chi_{2}^{2}\left[-9-40 \sqrt{15} \chi_{g}\right. \\
&\left.\left.\left.+60\left[9 \chi_{1}^{2}-2 \chi_{1}\left(\sqrt{15}-3 \chi_{g}\right)+\chi_{g}^{2}\right]\right]\right]\right] \\
&
\end{aligned}
$$

\section{References}

1. J.M. Maldacena, The large $N$ limit of superconformal field theories and supergravity. Adv. Theor. Math. Phys. 2, 231-252 (1998). arXiv:hep-th/9711200

2. C. Ahn, J. Paeng, Three-dimensional SCFTs, supersymmetric domain wall and renormalization group flow. Nucl. Phys. B 595, 119-137 (2001). arXiv:hep-th/0008065

3. C. Ahn, K. Woo, Supersymmetric domain wall and RG flow from 4-dimensional gauged $N=8$ supergravity. Nucl. Phys. B 599, 83-118 (2001). arXiv:hep-th/0011121

4. C. Ahn, T. Itoh, An $N=1$ supersymmetric $G_{2}$-invariant flow in M-theory. Nucl. Phys. B 627, 45-65 (2002). arXiv:hep-th/0112010

5. N. Bobev, N. Halmagyi, K. Pilch, N.P. Warner, Holographic, $N=1$ supersymmetric RG flows on M2 branes. JHEP 09, 043 (2009). arXiv:0901.2376

6. T. Fischbacher, K. Pilch, N.P. Warner, New supersymmetric and stable, non-supersymmetric phases in supergravity and holographic field theory. arXiv: 1010.4910

7. A. Guarino, On new maximal supergravity and its BPS domainwalls. JHEP 02, 026 (2014). arXiv:1311.0785

8. J. Tarrio, O. Varela, Electric/magnetic duality and RG flows in $A d S_{4} / C F T_{3}$. JHEP 01, 071 (2014). arXiv:1311.2933

9. Y. Pang, C.N. Pope, J. Rong, Holographic RG flow in a new $S O(3) \times S O(3)$ sector of $\omega$-deformed $S O(8)$ gauged $N=8$ supergravity. JHEP 08, 122 (2015). arXiv:1506.04270

10. J. Bagger, N. Lambert, Gauge symmetry and supersymmetry of multiple M2-branes. Phys. Rev. D 77, 065008 (2008). arXiv:0711.0955

11. O. Aharony, O. Bergman, D.L. Jafferis, J. Maldacena, $N=6$ superconformal Chern-Simons-matter theories, M2-branes and their gravity duals. JHEP 10, 091 (2008). arXiv:0806.1218

12. P. Karndumri, Holographic RG flows in $N=3$ Chern-Simonsmatter theory from $N=34$ D gauged supergravity. Phys. Rev. D 94, 045006 (2016). arXiv:1601.05703

13. P. Karndumri, K. Upathambhakul, Gaugings of four-dimensional $N=3$ supergravity and $\mathrm{AdS}_{4} / \mathrm{CFT}_{3}$ holography. Phys. Rev. D 93, 125017 (2016). arXiv: 1602.02254

14. P. Karndumri, Supersymmetric deformations of 3D SCFTs from tri-sasakian truncation. Eur. Phys. J. C 77, 130 (2017). arXiv: 1610.07983

15. E. Bergshoeff, I.G. Koh, E. Sezgin, Coupling of Yang-Mills to $N=4, d=4$ supergravity. Phys. Lett. B 155, 71-75 (1985)

16. M. de Roo, P. Wagemans, Gauged matter coupling in $N=4$ supergravity. Nucl. Phys. B 262, 644-660 (1985)

17. C. Horst, J. Louis, P. Smyth, Electrically gauged $N=4$ supergravities in $D=4$ with $N=2$ vacua. JHEP 03, 144 (2013). arXiv: 1212.4707

18. P. Wagemans, Breaking of $N=4$ supergravity to $N=1, N=2$ at $\Lambda=0$. Phys. Lett. B 206, 241 (1988)

19. J. Schon, M. Weidner, Gauged $N=4$ supergravities. JHEP 05, 034 (2006). arXiv:hep-th/0602024

20. G. Dibitetto, A. Guarino, D. Roest, Charting the landscape of $N=$ 4 flux compactifications. JHEP 03, 137 (2011). arXiv:1102.0239

21. J.P. Derendinger, C. Kounnas, P.M. Petropoulos, F. Zwirner, Superpotentials in IIA compactifications with general fluxes. Nucl. Phys. B 715, 211-233 (2005). arXiv:hep-th/0411276

22. O. DeWolfe, A. Giryavets, S. Kachru, W. Taylor, Type IIA moduli stabilization. JHEP 07, 066 (2005). arXiv:hep-th/0505160

23. G. Dall' Agata, G. Villadoro, F. Zwirner, Type-IIA flux compactifications and $\mathrm{N}=4$ gauged supergravities. JHEP 08, 018 (2009). arXiv:0906.0370

24. G. Villadoro, F. Zwirner, $N=1$ effective potential from dual typeIIA D6/O6 orientifolds with general fluxes. JHEP 06, 047 (2005). arXiv:hep-th/0503169 
25. U.H. Danielsson, G. Dibitetto, S.C. Vargas, Universal isolation in the AdS landscape. Phys. Rev. D 94, 126002 (2016). arXiv:1605.09289

26. N. Bobev, K. Pilch, N.P. Warner, Supersymmetric Janus solutions in four dimensions. JHEP 1406, 058 (2014). arXiv: 1311.4883

27. P. Karndumri, Supersymmetric Janus solutions in four-dimensional $N=3$ gauged supergravity. Phys. Rev. D 93, 125012 (2016). arXiv:1604.06007

28. G. Inverso, H. Samtleben, M. Trigiante, Type II origin of dyonic gaugings. Phys. Rev. D 95, 066020 (2017). arXiv: 1612.05123

29. D. Bak, M. Gutperle, S. Hirano, A dilatonic deformation of $A d S_{5}$ and its field theory dual. JHEP 05, 072 (2003). arXiv:hep-th/0304129

30. A. Clark, A. Karch, Super Janus. JHEP 10, 094 (2005). arXiv:hep-th/0506265
31. M.W. Suh, Supersymmetric Janus solutions in five and ten dimensions. JHEP 09, 064 (2011). arXiv:1107.2796

32. M. Gutperle, J. Samani, Holographic RG-flows and Boundary CFTs. Phys. Rev. D 86, 106007 (2012). arXiv:1207.7325

33. D.M. McAvity, H. Osborn, Conformal field theories near a boundary in general dimensions. Nucl. Phys. B 455, 522 (1995). arXiv:cond-mat/9505127

34. S.S. Gubser, Curvature singularities: the good, the bad and the naked. Adv. Theor. Math. Phys. 4, 679-745 (2000). arXiv:hep-th/0002160

35. J. Louis, H. Triendl, Maximally supersymmetric $A d S_{4}$ vacua in $N=4$ supergravity. JHEP 10, 007 (2014). arXiv:1406.3363

36. J. Maldacena, C. Nunez, Supergravity description of field theories on curved manifolds and a no go theorem. Int. J. Mod. Phys. A 16, 822 (2001). arXiv:hep-th/0007018 